\title{
Testing the Ginzburg-Landau approximation for three-flavor crystalline color superconductivity
}

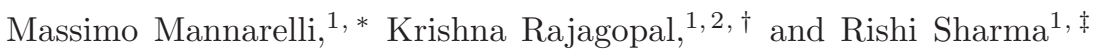 \\ ${ }^{1}$ Center for Theoretical Physics, Massachusetts Institute of Technology, Cambridge, MA 02139 \\ ${ }^{2}$ Nuclear Science Division, MS 70R319, Lawrence Berkeley National Laboratory, Berkeley, CA 94720
}

(Dated: April 11, 2018)

\begin{abstract}
It is an open challenge to analyze the crystalline color superconducting phases that may arise in cold dense, but not asymptotically dense, three-flavor quark matter. At present the only approximation within which it seems possible to compare the free energies of the myriad possible crystal structures is the Ginzburg-Landau approximation. Here, we test this approximation on a particularly simple "crystal" structure in which there are only two condensates $\langle u s\rangle \sim \Delta \exp \left(i \mathbf{q}_{2} \cdot \mathbf{r}\right)$ and $\langle u d\rangle \sim \Delta \exp \left(i \mathbf{q}_{\mathbf{3}} \cdot \mathbf{r}\right)$ whose position-space dependence is that of two plane waves with wave vectors $\mathbf{q}_{2}$ and $\mathbf{q}_{3}$ at arbitrary angles. For this case, we are able to solve the mean-field gap equation without making a Ginzburg-Landau approximation. We find that the Ginzburg-Landau approximation works in the $\Delta \rightarrow 0$ limit as expected, find that it correctly predicts that $\Delta$ decreases with increasing angle between $\mathbf{q}_{\mathbf{2}}$ and $\mathbf{q}_{\mathbf{3}}$ meaning that the phase with $\mathbf{q}_{\mathbf{2}} \| \mathbf{q}_{\mathbf{3}}$ has the lowest free energy, and find that the Ginzburg-Landau approximation is conservative in the sense that it underestimates $\Delta$ at all values of the angle between $\mathbf{q}_{2}$ and $\mathbf{q}_{3}$.
\end{abstract}

PACS numbers: $12.38 . \mathrm{Mh}, 24.85 .+\mathrm{p}$

\section{INTRODUCTION}

Quantum chromodynamics predicts that at densities that are high enough that baryons are crushed into quark matter, the quark matter that results features pairing between quarks at low enough temperatures, meaning that it is in one of a family of possible color superconducting phases 1]. The "laboratory" where color superconducting quark matter is most likely to be found is the interior of compact stars. Except during the first few seconds after their birth in supernovae, these stars have temperatures well below the tens of $\mathrm{MeV}$ critical temperature expected for color superconductivity, meaning that if these stars feature quark matter cores, these cores will be color superconductors.

The essence of color superconductivity is quark pairing, leading to Meissner effects for (some) color magnetic fields, driven by the BCS mechanism which operates whenever there are attractive interactions between fermions at a Fermi surface [2]. The interaction between quarks in QCD is strong and is attractive between quarks that are antisymmetric in color, so we expect cold dense quark matter to exhibit color superconductivity. We shall only consider Cooper pairs whose pair wave function is antisymmetric in Dirac indices - the relativistic generalization of zero total spin. (Other possibilities have been investigated [1, 3, 4, [5, 6] and found to be less favorable.) This in turn requires antisymmetry in flavor, meaning in particular that the two quarks in a Cooper pair must have different flavor.

\footnotetext{
*Electronic address: massimo@lns.mit.edu

${ }^{\dagger}$ Electronic address: krishna@lns.mit.edu

${ }^{\ddagger}$ Electronic address: sharma@mit.edu
}

It is by now well-established that at sufficiently high densities, where the up, down and strange quarks can be treated on an equal footing and the disruptive effects of the strange quark mass can be neglected, quark matter is in the color-flavor locked (CFL) phase, in which quarks of all three colors and all three flavors form conventional Cooper pairs with zero total momentum [1, 5], and all fermionic excitations are gapped. However, even at the very center of a compact star the quark number chemical potential $\mu$ cannot be much larger than 500 $\mathrm{MeV}$, meaning that the strange quark mass $M_{s}$ (which is density dependent, lying somewhere between its vacuum current mass of about $100 \mathrm{MeV}$ and constituent mass of about $500 \mathrm{MeV}$ ) cannot be neglected. Furthermore, bulk matter, as relevant for a compact star, must be in weak equilibrium and must be electrically and color neutral 6, 7, 8, 9, 10]. All these factors work to separate the Fermi momenta of the three different flavors of quarks, and thus disfavor the cross-species BCS pairing that characterizes the CFL phase. If we imagine beginning at asymptotically high densities and reducing the density, and suppose that CFL pairing is disrupted by the heaviness of the strange quark before color superconducting quark matter is superseded by baryonic matter, the CFL phase must be replaced by some phase of quark matter in which there is less, and less symmetric, pairing. The nature of this phase is not currently established.

Within a spatially homogeneous ansatz, the next phase down in density is the gapless CFL (gCFL) phase 11, 12, 13, 14, 15, 16, 17, 18. In this phase, quarks of all three colors and all three flavors still form ordinary Cooper pairs, with each pair having zero total momentum, but there are regions of momentum space in which certain quarks do not succeed in pairing, and these regions are bounded by momenta at which certain fermionic quasiparticles are gapless. This variation on BCS pairing - 
in which the same species of fermions that pair feature gapless quasiparticles - was first proposed for two flavor quark matter [19] and in an atomic physics context 20]. In all these contexts, however, the gapless paired state turns out in general to suffer from a "magnetic instability": it can lower its energy by the formation of counterpropagating currents [21, 22]. In the atomic physics context, the resolution of the instability is phase separation, into macroscopic regions of two phases in one of which standard BCS pairing occurs and in the other of which no pairing occurs 23, 24, 25]. In three-flavor quark matter, where the instability of the gCFL phase has been established in Refs. 22], phase coexistence would require coexisting components with opposite color charges, in addition to opposite electric charges, making it very unlikely that a phase separated solution can have lower energy than the gCFL phase 12, 13]. Furthermore, color superconducting phases which are less symmetric than the CFL phase but still involve only conventional BCS pairing, for example the much-studied 2SC phase in which only two colors of up and down quarks pair [4, 26, 27] but including also many other possibilities 28], cannot be the resolution of the gCFL instability [6, 28]. It seems likely, therefore, that a ground state with counter-propagating currents is required. This could take the form of a crystalline color superconductor 29, 30, 31, 32, 33, 34, 35, 36, 37, 38 - the QCD analogue of a form of non-BCS pairing first considered by Larkin, Ovchinnikov, Fulde and Ferrell [39]. Or, given that the CFL phase itself is likely augmented by kaon condensation [40, 41], it could take the form of a phase in which a CFL kaon condensate carries a current in one direction balanced by a counter-propagating current in the opposite direction carried by gapless quark quasiparticles [42]. This meson supercurrent phase has been shown to have a lower free energy than the gCFL phase. The investigation of crystalline color superconductivity in three-flavor QCD has only just begun 37]. Although such phases seem to be free from magnetic instability [38], it remains to be seen whether such a phase can have a lower free energy than the meson current phase, making it a possible resolution to the gCFL instability. The simplest "crystal" structures do not suffice, but experience in the two-flavor context [36] suggests that realistic crystal structures constructed from more plane waves will prove to be qualitatively more robust. Once we know (or have a well-motivated conjecture for) the favored crystal structure, the challenge will then be to calculate its shear modulus and the pinning force it exerts on rotational vortices trying to move through it, as these are the microphysical inputs needed to determine whether observations of pulsar glitches can be used to rule out (or in) the presence quark matter in the crystalline color superconducting phase within compact stars [29].

Determining the favored crystal structure(s) in the crystalline color superconducting phase(s) of three-flavor QCD requires determining the gaps and comparing the free energies for very many candidate structures, as there are even more possibilities than the many that were investigated in the two-flavor context [36]. As there, it seems likely that progress will require making a GinzburgLandau approximation. This approximation is controlled if $\Delta \ll \Delta_{0}$, where $\Delta$ is the gap parameter of the crystalline color superconducting phase itself and $\Delta_{0}$ is the gap parameter in the CFL phase that would occur if $M_{s}$ were zero. This approximation will be dubious precisely in the case of interest: if a crystalline phase exists with a free energy lower than that of the gCFL phase, such a phase will be characterized by robust pairing meaning that $\Delta$ will not be much smaller than $\Delta_{0}$. Our purpose in this paper is to analyze a particularly simple one parameter family of "crystal" structures in three-flavor quark matter, simple enough that we can do the analysis both with and without the Ginzburg-Landau approximation.

We shall work throughout in a Nambu-Jona-Lasinio (NJL) model in which the QCD interaction between quarks is replaced by a point-like four-quark interaction, with the quantum numbers of single-gluon exchange, analyzed in mean field theory. This is not a controlled approximation. However, it suffices for our purposes: because this model has attraction in the same channels as in QCD, its high density phase is the CFL phase; and, the Fermi surface splitting effects whose qualitative consequences we wish to study can all be built into the model. Note that we shall assume throughout that $\Delta_{0} \ll \mu$. This weak coupling assumption means that the pairing is dominated by modes near the Fermi surfaces, justifying the analysis of the model using High Density Effective Theory (HDET) [3, 44]. Thus, we shall be comparing two calculations: one making the Ginzburg-Landau assumption that $\Delta \ll \Delta_{0} \ll \mu$ and the other allowing for $\Delta \sim \Delta_{0} \ll \mu$. The Ginzburg-Landau calculation can be extended to more complicated crystal structures than the simple ones that we shall analyze. We shall find that the Ginzburg-Landau approximation works when it should and that, at least for the simple crystal structure we analyze, it is conservative in the sense that when it breaks down it always underestimates the gap $\Delta$ and the condensation energy. Furthermore, we find that the GinzburgLandau approximation correctly determines which crystal structure among the one parameter family that we analyze has the largest gap and lowest free energy. We shall see, however, that the range of validity of the GinzburgLandau approximation does depend on the crystal structure.

Our paper is organized as follows. In Section $\prod$ we shall describe the one parameter family of three-flavor crystalline color superconductors that we analyze. In Section IIII we present the results of our Ginzburg-Landau analysis. In Section IV we recast the High Density Effective Theory slightly, as needed for our purposes, and redo our analysis without the Ginzburg-Landau approximation. In Section $\nabla$ we show the numerical results of our analyses, make comparisons, and draw conclusions. 


\section{MODEL AND ANSATZ}

We shall analyze quark matter containing massless $u$ and $d$ quarks and $s$ quarks with an effective mass $M_{s}$. (Although the strange quark mass can be determined self-consistently by solving for an $\langle\bar{s} s\rangle$ condensate 9, 17, 18], we shall leave this to future work and treat $M_{s}$ as a parameter.) The Lagrangian density describing this system in the absence of interactions is given by

$$
\mathcal{L}_{0}=\bar{\psi}_{i \alpha}\left(i \not \partial_{i j}^{\alpha \beta}-M_{i j}^{\alpha \beta}+\mu_{i j}^{\alpha \beta} \gamma_{0}\right) \psi_{\beta j}
$$

where $i, j=1,2,3$ are flavor indices and $\alpha, \beta=1,2,3$ are color indices, where we have suppressed the Dirac indices, where $M_{i j}^{\alpha \beta}=\delta^{\alpha \beta} \operatorname{diag}\left(0,0, M_{s}\right)_{i j}$ is the mass matrix, where $\partial_{i j}^{\alpha \beta}=\partial \delta^{\alpha \beta} \delta_{i j}$ and where the quark chemical potential matrix is given by

$$
\mu_{i j}^{\alpha \beta}=\left(\mu \delta_{i j}-\mu_{e} Q_{i j}\right) \delta^{\alpha \beta}+\delta_{i j}\left(\mu_{3} T_{3}^{\alpha \beta}+\frac{2}{\sqrt{3}} \mu_{8} T_{8}^{\alpha \beta}\right)
$$

with $Q=\operatorname{diag}(2 / 3,-1 / 3,-1 / 3)_{i j}$ the quark electriccharge matrix and $T_{3}$ and $T_{8}$ the Gell-Mann matrices in color space. We shall quote results at quark number chemical potential $\mu=500 \mathrm{MeV}$ throughout.

In QCD, $\mu_{e}, \mu_{3}$ and $\mu_{8}$ are the zeroth components of electromagnetic and color gauge fields, and the gauge field dynamics ensure that they take on values such that the matter is neutral [6, 45], satisfying

$$
\frac{\partial \Omega}{\partial \mu_{e}}=\frac{\partial \Omega}{\partial \mu_{3}}=\frac{\partial \Omega}{\partial \mu_{8}}=0
$$

with $\Omega$ the free energy density of the system. In the NJL model that we shall employ, in which quarks interact via four-fermion interactions and there are no gauge fields, we introduce $\mu_{e}, \mu_{3}$ and $\mu_{8}$ by hand, and choose them to satisfy the neutrality constraints (3). The assumption of weak equilibrium is built into the calculation via the fact that the only flavor-dependent chemical potential is $\mu_{e}$, ensuring for example that the chemical potentials of $d$ and $s$ quarks with the same color must be equal. Because the strange quarks have greater mass, the equality of their chemical potentials implies that the $s$ quarks have smaller Fermi momenta than the $d$ quarks in the absence of BCS pairing. In the absence of pairing, then, because weak equilibrium drives the massive strange quarks to be less numerous than the down quarks, electrical neutrality requires a $\mu_{e}>0$, which makes the up quarks less numerous than the down quarks and introduces some electrons into the system. In the absence of pairing, color neutrality is obtained with $\mu_{3}=\mu_{8}=0$.

The Fermi momenta of the quarks and electrons in quark matter that is electrically and color neutral and in weak equilibrium are given in the absence of pairing by

$$
\begin{aligned}
& p_{F}^{d}=\mu+\frac{\mu_{e}}{3} \\
& p_{F}^{u}=\mu-\frac{2 \mu_{e}}{3} \\
& p_{F}^{s}=\sqrt{\left(\mu+\frac{\mu_{e}}{3}\right)^{2}-M_{s}^{2}} \approx \mu+\frac{\mu_{e}}{3}-\frac{M_{s}^{2}}{2 \mu} \\
& p_{F}^{e}=\mu_{e}
\end{aligned}
$$

where we have simplified $p_{F}^{s}$ upon assuming that $M_{s}$ and $\mu_{e}$ are small compared to $\mu$ by working only to linear order in $\mu_{e}$ and $M_{s}^{2}$. To this order, electric neutrality requires

$$
\mu_{e}=\frac{M_{s}^{2}}{4 \mu}
$$

yielding

$$
\begin{aligned}
& p_{F}^{d}=\mu+\frac{M_{s}^{2}}{12 \mu}=p_{F}^{u}+\frac{M_{s}^{2}}{4 \mu} \\
& p_{F}^{u}=\mu-\frac{M_{s}^{2}}{6 \mu} \\
& p_{F}^{s}=\mu-\frac{5 M_{s}^{2}}{12 \mu}=p_{F}^{u}-\frac{M_{s}^{2}}{4 \mu} \\
& p_{F}^{e}=\frac{M_{s}^{2}}{4 \mu} .
\end{aligned}
$$

We see from (4) that to leading order in $M_{s}^{2}$ and $\mu_{e}$, the effect of the strange quark mass on unpaired quark matter is as if instead one reduced the strange quark chemical potential by $M_{s}^{2} /(2 \mu)$. We shall make this approximation throughout. The corrections to this approximation in an NJL analysis of a two-flavor crystalline color superconductor have been evaluated and found to be small [33], and we expect the same to be true here. Upon making this assumption, we need no longer be careful about the distinction between $p_{F}$ 's and $\mu$ 's, as we can simply think of the three flavors of quarks as if they have chemical potentials

$$
\begin{aligned}
& \mu_{d}=\mu_{u}+2 \delta \mu_{3} \\
& \mu_{u}=p_{F}^{u} \\
& \mu_{s}=\mu_{u}-2 \delta \mu_{2}
\end{aligned}
$$

with

$$
\delta \mu_{3}=\delta \mu_{2}=\frac{M_{s}^{2}}{8 \mu} \equiv \delta \mu
$$

where the choice of subscripts indicates that $2 \delta \mu_{2}$ is the splitting between the Fermi surfaces for quarks 1 and 3 and $2 \delta \mu_{3}$ is that between the Fermi surfaces for quarks 1 and 2, identifying $u, d, s$ with $1,2,3$. (The prefactor 2 in the equations defining the $\delta \mu$ 's is chosen to agree with the notation used in the analysis of crystalline color superconductivity in a two flavor model [29], in which 
the two Fermi surfaces were denoted by $\mu \pm \delta \mu$ meaning that they were separated by $2 \delta \mu$.)

As described in Refs. 6, 9, 11, 46, BCS pairing introduces qualitative changes into the analysis of neutrality. For example, in the CFL phase $\mu_{e}=0$ and $\mu_{8}$ is nonzero and of order $M_{s}^{2} / \mu$. This arises because the construction of a phase in which BCS pairing occurs between fermions whose Fermi surface would be split in the absence of pairing can be described as follows. First, adjust the Fermi surfaces of those fermions that pair to make them equal. This costs a free energy price of order $\delta \mu^{2} \mu^{2}$. And, it changes the relation between the chemical potentials and the particle numbers, meaning that the $\mu$ 's required for neutrality can change qualitatively as happens in the CFL example. Second, pair. This yields a free energy benefit of order $\Delta_{0}^{2} \mu^{2}$, where $\Delta_{0}$ is the gap parameter describing the BCS pairing. Hence, BCS pairing will only occur if the attraction between the fermions is large enough that $\Delta_{0} \gtrsim \delta \mu$. In the CFL context, in which $\langle u d\rangle,\langle u s\rangle$ and $\langle d s\rangle$ pairing is fighting against the splitting between the $d, u$ and $s$ Fermi surfaces described above, it turns out that CFL pairing can occur if $\Delta_{0}>4 \delta \mu=M_{s}^{2} /(2 \mu)$ [1] , a criterion that is reduced somewhat by kaon condensation which acts to stabilize CFL pairing [41]. In this paper we are considering quark matter at densities that are low enough $\left(\mu<M_{s}^{2} /\left(2 \Delta_{0}\right)\right)$ that CFL pairing is not possible. The gap parameter $\Delta_{0}$ that would characterize the CFL phase if $M_{s}^{2}$ and $\delta \mu$ were zero is nevertheless an important scale in our problem, as it quantifies the strength of the attraction between quarks. Estimates of the magnitude of $\Delta_{0}$ are typically in the tens of $\mathrm{MeV}$, perhaps as large as 100 $\mathrm{MeV}$ 1]. We shall treat $\Delta_{0}$ as a parameter, and quote results for $\Delta_{0}=25 \mathrm{MeV}$.

Crystalline color superconductivity can be thought of as the answer to the question: "Is there a way to pair quarks at differing Fermi surfaces without first equalizing their Fermi momenta, given that doing so exacts a cost?" The answer is "Yes, but it requires Cooper pairs with nonzero total momentum." Ordinary BCS pairing pairs quarks with momenta $\mathbf{p}$ and $-\mathbf{p}$, meaning that if the Fermi surfaces are split at most one member of a pair can be at its Fermi surface. In the crystalline color superconducting phase, pairs with total momentum $2 \mathbf{q}$ condense, meaning that one member of the pair has momentum $\mathbf{p}+\mathbf{q}$ and the other has momentum $-\mathbf{p}+\mathbf{q}$ for some $\mathbf{p}[29,39]$. Suppose for a moment that only $u$ and $d$ quarks pair, making the analyses of a two-flavor model found in Refs. 29, 30, 31, 32, 33, 34, 35, 36. (and really going back to Ref. [39]) valid. We sketch the results of this analysis in the following two paragraphs.

The simplest "crystalline" phase is one in which only pairs with a single $\mathbf{q}$ condense, yielding a condensate

$$
\left\langle\psi_{u}(x) C \gamma_{5} \psi_{d}(x)\right\rangle \propto \Delta \exp (-2 i \mathbf{q} \cdot \mathbf{x})
$$

that is modulated in space like a plane wave. Assuming that $\Delta \ll \delta \mu \ll \mu$, the energetically favored value of $|\mathbf{q}| \equiv q$ turns out to be $q=\eta \delta \mu$, where the propor- tionality constant $\eta$ is given by $\eta=1.1997$ [29, 39]. If $\eta$ were 1 , then the only choice of $\mathbf{p}$ for which a Cooper pair with momenta $(-\mathbf{p}+\mathbf{q}, \mathbf{p}+\mathbf{q})$ would describe two quarks each on their respective Fermi surfaces would correspond to a quark on the north pole of one Fermi surface and a quark on the south pole of the other. Instead, with $\eta>1$, the quarks on each Fermi surface that can pair lie on one ring on each Fermi surface, the rings having opening angle $\psi_{0}=2 \cos ^{-1}(1 / \eta)=67.1^{\circ}$. The energetic calculation that determines $\eta$ can be thought of as balancing the gain in pairing energy as $\eta$ is increased beyond 1, allowing quarks on larger rings to pair, against the kinetic energy cost of Cooper pairs with greater total momentum. If the $\Delta / \delta \mu \rightarrow 0$ Ginzburg-Landau limit is not assumed, the pairing rings change from circular lines on the Fermi surfaces into ribbons of thickness $\sim \Delta$ and angular extent $\sim \Delta / \delta \mu$. The condensate (9) carries a current, which is balanced by a counter-propagating current carried by the unpaired quarks near their Fermi surfaces that are not in the pairing ribbons. Hence, the state carries no net current.

After solving a gap equation for $\Delta$ and then evaluating the free energy of the phase with condensate (9), one finds that this simplest "crystalline" phase is favored over twoflavor quark matter with either no pairing or BCS pairing only within a narrow window

$$
0.707<\frac{\delta \mu}{\Delta_{0}}<0.754 .
$$

At the upper boundary of this window, $\Delta \rightarrow 0$ and one finds a second order phase transition between the crystalline and unpaired phases. At the lower boundary, there is a first order transition between the crystalline and BCS paired phases. The crystalline phase persists in the weak coupling limit only if $\delta \mu / \Delta_{0}$ is held fixed, within the window (10), while the standard weakcoupling limit $\Delta_{0} / \mu \rightarrow 0$ is taken. Looking ahead to our context, and recalling that in three-flavor quark matter $\delta \mu=M_{s}^{2} /(8 \mu)$, we see that at high densities one finds the CFL phase (which is the three-flavor quark matter BCS phase) and in some window of lower densities one finds a crystalline phase. In the vicinity of the second order transition, where $\Delta \rightarrow 0$ and in particular where $\Delta / \delta \mu \rightarrow 0$ and, consequently given (10), $\Delta / \Delta_{0} \rightarrow 0$ a Ginzburg-Landau expansion of the free energy order by order in powers of $\Delta$ is controlled. Analysis within an NJL model shows that the results for $\Delta(\delta \mu)$ become accurate in the limit $\delta \mu \rightarrow 0.754 \Delta_{0}$ where $\Delta \rightarrow 0$, as must be the case, and show that the Ginzburg-Landau approximation underestimates $\Delta(\delta \mu)$ at all $\delta \mu$ [29, 36].

The Ginzburg-Landau analysis can then be applied to more complicated crystal structures in which Cooper pairs with several different q's, all with the same length but pointing in different directions, arise [36]. This analysis indicates that a face-centered cubic structure constructed as the sum of eight plane waves with q's pointing at the corners of a cube is favored, but it does not permit a quantitative evaluation of $\Delta(\delta \mu)$ in this case 
because it predicts a strong first order phase transition between the crystalline and unpaired phase, at some $\delta \mu$ significantly larger than $0.754 \Delta_{0}$, meaning that there is no value of $\delta \mu$ at which the Ginzburg-Landau approximation is under control [36].

Our purpose in this paper is to analyze a three-flavor analogue of the simplest "crystalline" condensate (9), with and without making the Ginzburg-Landau approximation $\Delta \ll \Delta_{0}$. We shall assume weak coupling, namely $\Delta_{0} \ll \mu$, throughout.

The analysis of neutrality in three-flavor quark matter in a crystalline color superconducting phase is very simple in the Ginzburg-Landau limit in which $\Delta \ll \delta \mu$ : because the construction of this phase does not involve rearranging any Fermi momenta prior to pairing, and because the assumption $\Delta \ll \delta \mu$ implies that the pairing does not significantly change any number densities, neutrality is achieved with the same chemical potentials $\mu_{e}=M_{s}^{2} /(4 \mu)$ and $\mu_{3}=\mu_{8}=0$ as in unpaired quark matter, and with Fermi momenta given in Eqs. (4), (6), and (77) as in unpaired quark matter. This result is correct only in the Ginzburg-Landau limit, but we expect that deviations from it in our NJL analysis are small, and so we shall fix the chemical potentials as in unpaired quark matter throughout this paper.

We consider a condensate of the form,

$$
\left\langle\psi_{\alpha i}(x) C \gamma_{5} \psi_{\beta j}(x)\right\rangle \propto \sum_{I=1}^{3} \Delta_{I} e^{-2 i \mathbf{q}_{\mathbf{I}} \cdot \mathbf{r}} \epsilon_{I \alpha \beta} \epsilon_{I i j},
$$

where $\mathbf{q}_{\mathbf{1}}, \mathbf{q}_{\mathbf{2}}$ and $\mathbf{q}_{\mathbf{3}}$ and $\Delta_{1}, \Delta_{2}$ and $\Delta_{3}$ are the wave vectors and gap parameters describing pairing between the $(d, s),(u, s)$ and $(u, d)$ quarks respectively, whose Fermi momenta are split by $2 \delta \mu_{1}, 2 \delta \mu_{2}$ and $2 \delta \mu_{3}$ respectively. From (7), we see that $\delta \mu_{2}=\delta \mu_{3}=$ $\delta \mu_{1} / 2=M_{s}^{2} /(8 \mu)$. The condensate (11), first analyzed in Ref. 37], is the natural generalization of the condensate (9) to the three-flavor setting, and the natural generalization of the CFL condensate (obtained by setting $\left.\mathbf{q}_{1}=\mathbf{q}_{2}=\mathbf{q}_{3}=\mathbf{0}\right)$ to the crystalline color superconductor setting.

We shall make the further simplifying assumption that $\Delta_{1}=0$. Given that $\delta \mu_{1}$ is twice $\delta \mu_{2}$ or $\delta \mu_{3}$, it seems reasonable that $\Delta_{1} \ll \Delta_{2}, \Delta_{3}$. We leave a quantitative investigation of the size of $\Delta_{1}$ to future work, however. With $\Delta_{1}$ set to zero, the symmetry of the problem is such that we expect and find solutions with $\Delta_{2}=\Delta_{3} \equiv \Delta$. It should be noted, however, that these simplifications are rigorous only in the Ginzburg-Landau limit in which $\Delta \ll \delta \mu$. A full investigation of cases in which $\Delta \sim \delta \mu$ requires investigating the Ginzburg-Landau results $\mu_{3}=$ $\mu_{8}=0, \mu_{e}=M_{s}^{2} /(4 \mu), \Delta_{1}=0$, and $\Delta_{2}=\Delta_{3}=\Delta$ anew, as beyond the Ginzburg-Landau approximation these are all assumptions, not results.

In an NJL model in which the quarks interact via a point-like four fermion interaction, analyzed at the mean field level, if we assume that the only condensate is that in Eq. (11) with $\Delta_{1}=0$ the interaction term added to the Lagrangian (11) can be written simply as

$$
\mathcal{L}_{\Delta}=\sum_{I=2}^{3} \Delta_{I}^{*} e^{i 2 \mathbf{q} \cdot \mathbf{r}} \epsilon_{I \alpha \beta} \epsilon_{I i j} \psi_{i \alpha} C \gamma_{5} \psi_{\beta j}+\text { h.c. }
$$

Note that since the direction of one of the two wave vectors is arbitrary, the quantities that have to be determined by minimizing the free-energy are $\Delta$, the magnitude of the two wave vectors, and the angle $\phi$ between $\hat{\mathbf{q}}_{2}$ and $\hat{\mathbf{q}}_{3}$. We shall see in the next section that, as in the two-flavor model, the magnitude of the wave vectors is given in the Ginzburg-Landau approximation by $\left|\mathbf{q}_{2}\right|=\left|\mathbf{q}_{3}\right|=\eta \delta \mu$ with $\eta=1.1997$. And, we shall see that, again as in the two-flavor model, $\Delta \rightarrow 0$ at $\delta \mu \rightarrow 0.754 \Delta_{0}$, corresponding to $M_{s}^{2} / \mu=6.032 \Delta_{0}$. In the vicinity of this second order phase transition, the Ginzburg-Landau approximation is controlled, as in the two-flavor model. However, the angle $\phi$ between the wave vectors is a degree of freedom present here that has no analogue in the two-flavor model with condensate (9). We shall determine it within the Ginzburg-Landau approximation in the next section, and check this result in Section IV.

\section{GINZBURG-LANDAU ANALYSIS}

The free energy of the crystalline color superconducting phase can only depend on the gaps $\Delta_{I}$ through $\left|\Delta_{I}\right|^{2}$, because the Lagrangian has $U(1)$ symmetries associated with $u-, d$ - and $s$-quark number conservation. Of course, the condensate (11) breaks these symmetries, but it does so spontaneously. Hence, when the free energy for the three-flavor crystalline color superconductor is expanded in powers of the $\Delta_{I}$ 's, the only terms that can arise up to fourth order take the form $\left|\Delta_{I}\right|^{2},\left|\Delta_{I}\right|^{4}$, and $\left|\Delta_{I}\right|^{2}\left|\Delta_{J}\right|^{2}$ for $I \neq J$. As we are setting $\Delta_{1}=0$, this means that the Ginzburg-Landau expansion of the free energy can be written

$\Omega=\Omega_{n}+\frac{\alpha}{2}\left(\Delta_{2}^{2}+\Delta_{3}^{2}\right)+\frac{\beta}{4}\left(\Delta_{2}^{4}+\Delta_{3}^{4}\right)+\frac{\beta_{23}}{2} \Delta_{2}^{2} \Delta_{3}^{2}+\mathcal{O}\left(\Delta^{6}\right)$,

where we have dropped the absolute value signs as henceforth we will assume that the $U(1)$ symmetries are broken such as to give real $\Delta$ 's. The contribution $\Omega_{n}$ is the free energy for unpaired ("normal") neutral quark matter with $\mu_{3}=\mu_{8}=0$ and $\mu_{e}=M_{s}^{2} /(4 \mu)$. Recall that $\Delta_{2}$ describes up-strange pairs with momentum $2 \mathbf{q}_{2}, \Delta_{3}$ describes up-down pairs with momentum $2 \mathbf{q}_{3}$, and the three Fermi surfaces are ordered $d$ then $u$ then $s$ from biggest to smallest, each separated from the next by $2 \delta \mu=M_{s}^{2} /(4 \mu)$. The Ginzburg-Landau expansion is controlled in the limit in which $\Delta_{2} / \delta \mu \rightarrow 0$ and $\Delta_{3} / \delta \mu \rightarrow 0$. In the present context, it was first analyzed in Ref. 37]. The coefficients in the Ginzburg-Landau 
expansion (13) are given by the expressions

$$
\begin{aligned}
\alpha & =\frac{4 \mu^{2}}{\pi^{2}}\left(-1+\frac{\delta \mu}{2 q} \ln \left|\frac{\delta \mu+q}{\delta \mu-q}\right|+\frac{1}{2} \ln \left|\frac{4\left(\delta \mu^{2}-q^{2}\right)}{\Delta_{0}^{2}}\right|\right), \\
\beta & =\frac{\mu^{2}}{2 \pi^{2}} \Re e \int \frac{d \hat{\mathbf{p}}}{4 \pi} \frac{-1}{(i \epsilon-\hat{\mathbf{p}} \cdot \mathbf{q}+\delta \mu)^{2}}=\frac{\mu^{2}}{\pi^{2}} \frac{1}{q^{2}-\delta \mu^{2}}, \\
\beta_{23} & =\frac{\mu^{2}}{2 \pi^{2}} \Re e \int \frac{d \hat{\mathbf{p}}}{4 \pi} \frac{-1}{\left(i \epsilon-\hat{\mathbf{p}} \cdot \mathbf{q}_{2}-\delta \mu\right)\left(i \epsilon-\hat{\mathbf{p}} \cdot \mathbf{q}_{3}+\delta \mu\right)},
\end{aligned}
$$

where $q \equiv|\mathbf{q}|$. Note that in (13) the coefficients $\alpha$ and $\beta$ multiply terms involving only $\Delta_{2}$ or only $\Delta_{3}$. Since $\Delta_{2}$ and $\Delta_{3}$ separately each describe pairing between two fermions with Fermi momenta separated by $2 \delta \mu$, this means that the expressions for $\alpha$ and $\beta$ are exactly as derived in the two-flavor analysis of Ref. [36]. The coefficient $\beta_{23}$, derived in Ref. [37], is instead characteristic of the 3-flavor case and represents the interaction term between the two different condensates. This is the only term in which a dependence of the free energy on the angle $\phi$ between $\hat{\mathbf{q}}_{2}$ and $\hat{\mathbf{q}}_{3}$ can arise. Note that we have written explicitly the $i \epsilon$ prescription needed to evaluate the integral expressions for both $\beta$ and $\beta_{23}$, which we have determined by following carefully the derivation of (13) from the Ginzburg-Landau expansion of the Green's function in the mean field approximation [36]. The $\beta_{23^{-}}$ integrand in Eq. (14) has singular points, making locating its poles correctly in the complex plane crucial for the evaluation of $\beta_{23}$.

To quadratic order, the free energy (13) includes no interaction between the condensates $\Delta_{2}$ and $\Delta_{3}$, and so is independent of $\phi$. The expression for $\alpha$ can be analyzed as in Ref. [36]. As long as $\beta$ and $\beta_{23}$ are positive, as we shall see below is the case, there is a second order phase transition from unpaired quark matter to a phase with nonzero $\Delta_{2}$ and $\Delta_{3}$ at the largest value of $\delta \mu$ for which $\alpha=0$ for some value of $q$. For larger $\delta \mu, \alpha>0$ for all $q$ and the unpaired phase is stable. The transition occurs at $\delta \mu=0.754 \Delta_{0}$, where modes with $q=\eta \delta \mu$ for $\eta=1.1997$ become unstable to condensation, and $\Delta_{2}$ and $\Delta_{3}$ become nonzero. At lower values of $\delta \mu$, modes in a band of $q$ have $\alpha<0$ making them unstable, but the mode with $q=\eta \delta \mu$ is the one with the most negative $\alpha$ and we therefore assume that the condensate involves only the modes with $q_{2}=q_{3}=\eta \delta \mu$.

To evaluate the magnitude of $\Delta_{2}$ and $\Delta_{3}$ below the second order phase transition where they become nonzero, we need the quartic coefficients $\beta$ and $\beta_{23}$. We see that although the location of the second order phase transition is independent of $\phi$, the magnitude of the condensates below the transition can depend on $\phi$ through $\beta_{23}$. It is convenient to factor out the dependence of $\beta$ and $\beta_{23}$ on $\delta \mu$ and $\mu$. For $q=1.1997 \delta \mu$, then,

$$
\beta=2.276 \frac{\mu^{2}}{\pi^{2} \delta \mu^{2}}
$$

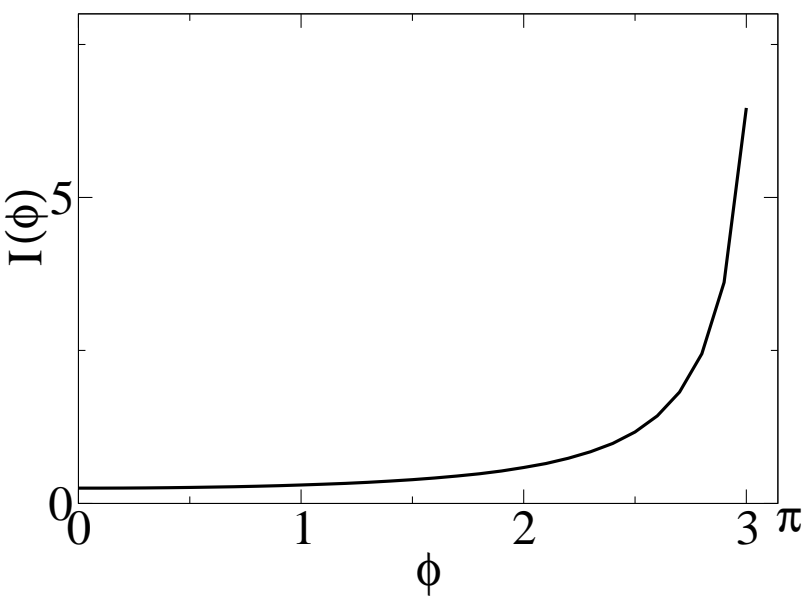

FIG. 1: $I(\phi)$, defined in Eq. (17), versus the angle $\phi$ between the wave vectors $\mathbf{q}_{2}$ and $\mathbf{q}_{3}$.

and, similarly, we write

$$
\beta_{23}=\frac{2 \mu^{2}}{\pi^{2} \delta \mu^{2}} I\left(\hat{\mathbf{q}}_{2}, \hat{\mathbf{q}}_{3}\right)
$$

defining

$I\left(\hat{\mathbf{q}}_{2}, \hat{\mathbf{q}}_{3}\right)=\Re e \int \frac{d \hat{p}}{4 \pi} \frac{-1}{\left(i \epsilon-\eta \hat{\mathbf{p}} \cdot \hat{\mathbf{q}}_{2}-1\right)\left(i \epsilon-\eta \hat{\mathbf{p}} \cdot \hat{\mathbf{q}}_{3}+1\right)}$

From rotational invariance, $I\left(\hat{\mathbf{q}}_{2}, \hat{\mathbf{q}}_{3}\right)$ depends only on $\phi$, the angle between $\hat{\mathbf{q}}_{2}$ and $\hat{\mathbf{q}}_{3}$. $I(\phi)$ can be evaluated numerically and the result is plotted in Fig 1 We note that $I(\phi)$ is an increasing function of $\phi$ and diverges at $\phi=\pi$, i.e. when the two $\mathbf{q}$ vectors are antiparallel. The minimum value of $I$ occurs at $\phi=0$, for parallel $\mathbf{q}$ vectors.

We now look for minima of the free energy with $\Delta_{2}=$ $\Delta_{3}=\Delta$, where the free energy can then be written as

$$
\Omega=\Omega_{n}+\alpha \Delta^{2}+\frac{1}{2}\left(\beta+\beta_{23}\right) \Delta^{4} .
$$

The expression for $\alpha$ in Eq. (14) yields the result that for $q=\eta \delta \mu, \alpha<0$ for $\delta \mu<0.7544 \Delta_{0}$, corresponding to $M_{s}^{2} / \mu<6.035 \Delta_{0} \simeq 151 \mathrm{MeV}$, where we have taken $\Delta_{0}=25 \mathrm{MeV}$. For values of $\delta \mu$ where $\alpha$ is negative, the solution is found at

$$
\Delta^{2}=\frac{|\alpha|}{\beta+\beta_{23}}
$$

with

$$
\Omega=\Omega_{n}-\frac{\alpha^{2}}{2\left(\beta+\beta_{23}\right)}
$$

These expressions can be evaluated using $\alpha, \beta$ and $\beta_{23}$ from Eqs. (14), (15) and (16) respectively. Since $\beta+$ $\beta_{23}$ is positive for all choices of the angle $\phi$, we see that 

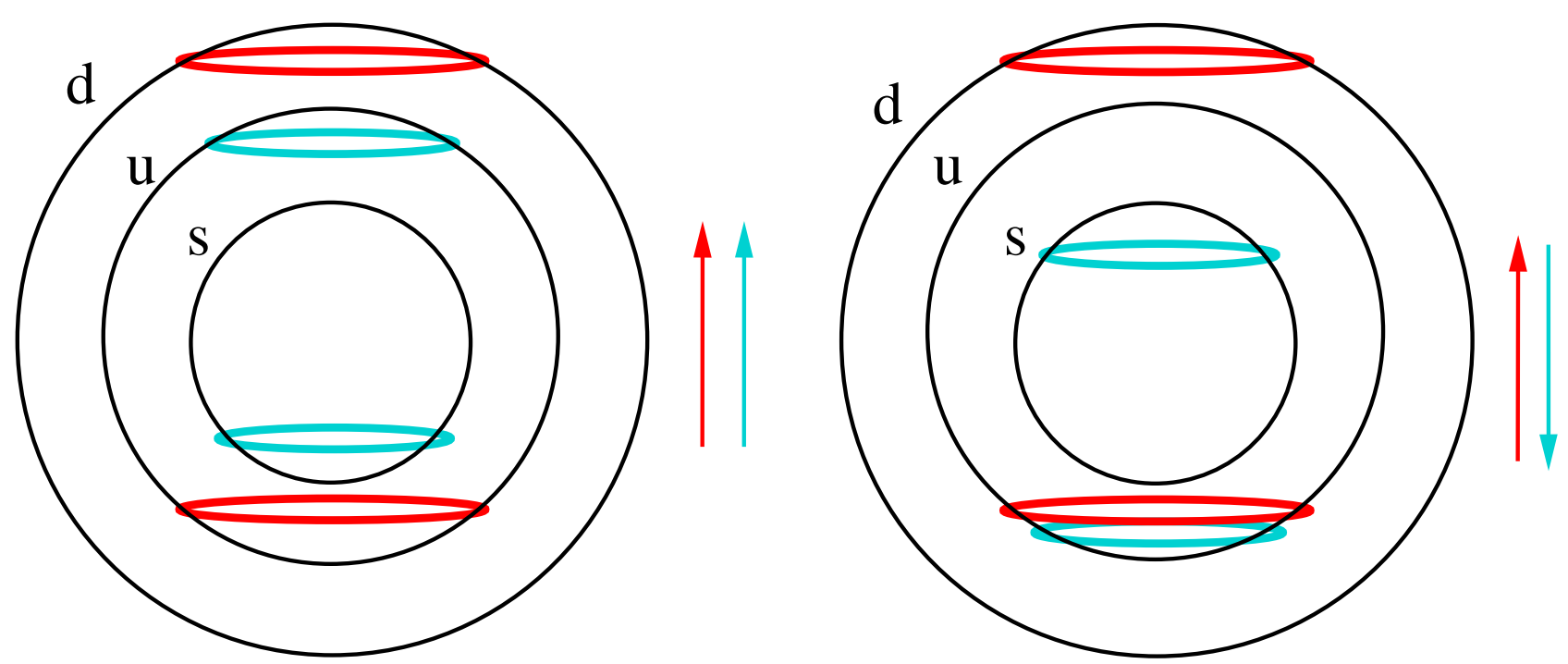

FIG. 2: Sketch showing where on the Fermi surfaces pairing occurs for condensates in which $\mathbf{q}_{\mathbf{2}}$ and $\mathbf{q}_{\mathbf{3}}$ are parallel (left panel) or antiparallel (right panel). The dark grey (red online) rings on the $d$ and $u$ Fermi surfaces indicate those quarks that contribute the most to the $\langle u d\rangle$ condensate with gap parameter $\Delta_{3}$ and wave vector $\mathbf{q}_{\mathbf{3}}$, which points upward in both panels. The light grey (teal online) rings on the $u$ and $s$ Fermi surfaces indicate those quarks that contribute most to the $\langle u s\rangle$ condensate with gap parameter $\Delta_{2}$ and wave vector $\mathbf{q}_{2}$, which points upward in the left panel and downward in the right panel. For illustrative purposes, we have greatly exaggerated the splitting between the Fermi surfaces, $\delta \mu / \mu$ relative to the values that we shall employ in the calculations reported in Section V.

the transition from the unpaired state to the crystalline phase with the simple "crystal" structure that we have employed is second order for all choices of $\phi$. The best choice of $\phi$ is the one that minimizes $\Omega$, meaning the

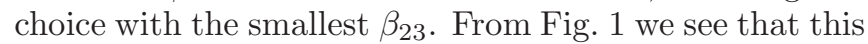
corresponds to $\phi=0$, with $\mathbf{q}_{2} \| \mathbf{q}_{3}$. We shall provide plots of $\Delta$ and $\Omega$ versus $M_{s}^{2} / \mu$ for various values of $\phi$ in Section V, where we shall compare these results to results obtained without making the Ginzburg-Landau approximation.

The free energy (13) can also be used to analyze the free energy of a two-flavor crystalline phase with a singleplane wave "crystal structure" in three flavor quark matter. Setting $\Delta_{2}=0$ and $\Delta_{3}=\Delta$ (or equivalently setting $\Delta_{3}=0$ and $\left.\Delta_{2}=\Delta\right)$ we find a solution with $\Delta^{2}=|\alpha| / \beta$ and $\Omega-\Omega_{n}=-\alpha^{2} /(4 \beta)$. Like the solution with $\Delta_{2}=\Delta_{3}$, this solution is neutral in the Ginzburg-Landau limit. The solution with $\Delta_{2}=\Delta_{3}$ has a lower free energy than that with $\Delta_{2}=0$ if $\left(\beta+\beta_{23}\right)<2 \beta$, meaning $\beta_{23}<\beta$. One can generalize to other solutions, and rule them out, by writing $\left(\Delta_{2}, \Delta_{3}\right)$ as $\left(\Delta_{r} \sin \theta, \Delta_{r} \cos \theta\right)$ and then rewriting Eq. (13) as

$$
\Omega=\Omega_{n}+\frac{\alpha}{2} \Delta_{r}^{2}+\frac{\beta}{4} \Delta_{r}^{4}+\left(\frac{\beta_{23}-\beta}{8}\right) \Delta_{r}^{2} \sin ^{2}(2 \theta) .
$$

As long as $\alpha<0$, there is a minimum with $\theta=\pi / 4$ (namely $\Delta_{2}=\Delta_{3}$ ) if $\beta_{23}<\beta$ and minima at $\theta=0$ and $\theta=\pi / 2$ if $\beta_{23}>\beta$. From Eqs. (15) and (16) we see that $\beta_{23}<\beta$ if $I(\phi)<1.138$, and from Fig. 1 we see that this occurs for $\phi<2.485$ radians, or $\phi<142.4^{\circ}$.

Although the divergence of $\beta_{23}$ at $\phi=\pi$ is not physically relevant, since for $\beta_{23}>\beta$ the lowest free energy solution has $\Delta_{2}$ or $\Delta_{3}$ vanishing, it is still worth gaining a qualitative understanding of the origin of the divergence. We see in Fig. 22 that there are two pairing rings on the up quark Fermi surface, because some up quarks pair with down quarks forming Cooper pairs with wave vector $2 \mathbf{q}_{3}$ and other up quarks pair with strange quarks forming Cooper pairs with wave vector $2 \mathbf{q}_{\mathbf{2}}$. However, as shown in the right panel of Fig. 2] if $\phi=\pi$ the two pairing rings on the up quark Fermi surface are close to coincident. In the weak-coupling limit in which $\delta \mu / \mu \rightarrow 0$ (and $\Delta_{0} \rightarrow 0$ with $\delta \mu / \Delta_{0}$ fixed) these two rings become precisely coincident. We attribute the divergence in $\beta_{23}$, which indicates that antiparallel wave vectors pay an infinite free energy price and hence are forbidden, to the coincidence of these two pairing rings. Loosely speaking, it is as if these up quarks do not know whether to pair with their putative strange or down partners and so do neither. In contrast, if $\phi=0$ as in the left panel of the Figure, the two pairing rings on the up Fermi surface are as far apart as they can be, and $\beta_{23}$ and the free energy of the state are minimized. This qualitative understanding also highlights that it is only in the strict Ginzburg-Landau and weak coupling limits that the cost of choosing antiparallel wave vectors diverges. If $\Delta / \delta \mu$ is small but nonzero, the pairing regions are ribbons on the Fermi surfaces instead of lines. And, if $\delta \mu / \mu$ is small but 
not taken to zero (as of course is the case in Fig. 2) then the two ribbons on the up Fermi surface will have slightly different diameter, as the Figure indicates. This means that we expect that if we do a calculation at small but nonzero $\Delta_{0} \sim \delta \mu$, and do not make a Ginzburg-Landau expansion, we should find some free energetic penalty for choosing $\phi=\pi$, but not a divergent one. We shall set up this calculation in Section IV and see this expectation confirmed in Section V.

\section{NJL ANALYSIS WITHOUT GINZBURG-LANDAU APPROXIMATION}

The two-flavor crystalline color superconducting phase with a single plane-wave "crystal" structure (9) has been analyzed in a variety of ways without making a GinzburgLandau approximation, going back to the work of Fulde and Ferrell 39]. In the QCD context, it was analyzed using a variational method in Ref. [29], using a diagrammatic method employing a modification of the NambuGorkov formalism in Refs. [30, 33], and using the NambuGorkov formalism simplified via the High Density Effective Theory in Ref. [31].

In the conventional Nambu-Gorkov formalism as applied to ordinary BCS pairing, one defines an eightcomponent Nambu-Gorkov spinor

$$
\Psi(\mathbf{p})=\left(\begin{array}{l}
\psi(\mathbf{p}) \\
\bar{\psi}^{T}(-\mathbf{p})
\end{array}\right)
$$

such that in this basis the pairing between fermions with momentum $\mathbf{p}$ and $\mathbf{- p}$ is described by an off-diagonal term in the fermion propagator. The condensate (9), however, describes pairing between $u$ quarks with momentum $\mathbf{p}+\mathbf{q}$ and $d$ quarks with momentum $-\mathbf{p}+\mathbf{q}$. This could be described via a propagator with terms in it that are off-diagonal in momentum space, rather than merely off-diagonal in "Nambu-Gorkov space". However, it is much easier to change to a basis in which the NambuGorkov spinor is written as [30]

$$
\Psi(\mathbf{p})=\left(\begin{array}{l}
\psi_{u}(\mathbf{p}+\mathbf{q}) \\
\psi_{d}(\mathbf{p}-\mathbf{q}) \\
\bar{\psi}_{u}^{T}(-\mathbf{p}-\mathbf{q}) \\
\bar{\psi}_{d}^{T}(-\mathbf{p}+\mathbf{q})
\end{array}\right)
$$

The pair condensate is then described by terms in the fermion propagator that are off-diagonal in NambuGorkov space, occurring in the $\psi_{u}-\bar{\psi}_{d}^{T}$ and $\psi_{d}-\bar{\psi}_{u}^{T}$ entries. In this basis the fermions that pair have $\mathbf{p}$ and $-\mathbf{p}$, making the propagator diagonal in $\mathbf{p}$-space and the calculation tractable. One must always keep in mind that it is $\mathbf{p}+\mathbf{q}$ and $-\mathbf{p}+\mathbf{q}$ that are the momenta of the fermions that pair, not $\mathbf{p}$ and $-\mathbf{p}$. The variable $\mathbf{p}$ is an integration variable: in the gap equation or in the expression for the free energy, integrating over $\mathbf{p}$ sums the contributions of all the fermions although of course it turns out that only those lying near ribbons on the Fermi surfaces contribute significantly. Since $\mathbf{p}$ is an integration variable, we are free to change variables, for example rewriting the Nambu-Gorkov spinor as

$$
\Psi(\mathbf{p})=\left(\begin{array}{l}
\psi_{u}(\mathbf{p}) \\
\psi_{d}(\mathbf{p}-2 \mathbf{q}) \\
\bar{\psi}_{u}^{T}(-\mathbf{p}) \\
\bar{\psi}_{d}^{T}(-\mathbf{p}+2 \mathbf{q})
\end{array}\right)
$$

The form of the Nambu-Gorkov spinor (24) immediately suggests that we analyze our three-flavor crystalline phase with condensate (11) with $\Delta_{1}$ set to zero by introducing the Nambu-Gorkov spinor

$$
\Psi(\mathbf{p})=\left(\begin{array}{l}
\psi_{u}(\mathbf{p}) \\
\psi_{d}\left(\mathbf{p}-2 \mathbf{q}_{3}\right) \\
\psi_{s}\left(\mathbf{p}-2 \mathbf{q}_{2}\right) \\
\bar{\psi}_{u}^{T}(-\mathbf{p}) \\
\bar{\psi}_{d}^{T}\left(-\mathbf{p}+2 \mathbf{q}_{3}\right) \\
\bar{\psi}_{s}^{T}\left(-\mathbf{p}+2 \mathbf{q}_{2}\right)
\end{array}\right) .
$$

Furthermore, it also indicates that it will not be possible to use this method of calculation if $\Delta_{1}$ were kept nonzero, except for the special case in which $\mathbf{q}_{1}=\mathbf{q}_{2}-\mathbf{q}_{3}$. (That is, except in this special case which is far from sufficiently generic, it will not be possible to choose a Nambu-Gorkov basis such that one obtains a propagator that is diagonal in some momentum variable p.) It is thus fortunate that, as explained in Section II, it is reasonable on physical grounds to begin with $\Delta_{1}=0$, as we do throughout this paper. Finally, it seems unlikely that this method can be employed to analyze more complicated crystal structures analogous to the face-centered cubic structure that is favored in the two-flavor case [36]. Investigating such possibilities is feasible in the Ginzburg-Landau approximation, but the condensate that we are analyzing, with $\Delta_{1}=0$ and $\Delta_{2}$ and $\Delta_{3}$ each multiplying a single plane wave, is the most complex example that we currently know how to analyze without making the Ginzburg-Landau approximation.

We now implement the calculation in the basis (25) using the High Density Effective Theory formalism of Refs. [31, 44], valid in the weak-coupling limit in which $\Delta_{0} \ll \mu$. We Fourier decompose the fermionic fields in the following nonstandard fashion:

$$
\psi_{i \alpha}(x)=e^{-i \mathbf{k}_{\mathbf{i}} \cdot \mathbf{x}} \int \frac{d \mathbf{n}}{4 \pi} e^{-i \mu \mathbf{n} \cdot \mathbf{x}}\left(\psi_{i \alpha, \mathbf{n}}(x)+\psi_{i \alpha, \mathbf{n}}^{-}(x)\right),
$$

where $\mathbf{n}$ is a unit three-vector whose direction is integrated over, where $\mathbf{k}_{\mathbf{i}}$ are three fixed vectors, one for each flavor, that we shall specify momentarily and where $\psi_{i \alpha, \mathbf{n}}(x)$ (resp. $\left.\psi_{i \alpha, \mathbf{n}}^{-}(x)\right)$ are positive (resp. negative) energy projections of the fermionic fields with flavor $i$ and color $\alpha$, as defined in Refs. [31, 44]. In the usual HDET approximation [4], the vectors $\mathbf{k}_{i}$ are zero and the field $\psi_{i \alpha, \mathbf{n}}(x)$ is used to describe quarks in a patch in momentum space in the vicinity of momentum $\mathbf{p}=\mu \mathbf{n}$. The 
introduction of the shift vectors means that now $\psi_{i \alpha, \mathbf{n}}(x)$ describes quarks with momenta in a patch in the vicinity of momentum $\mu \mathbf{n}+\mathbf{k}_{i}$, with $\mathbf{k}_{i}$ different for different flavors. To reproduce [25], then, it appears that we should choose

$$
\begin{aligned}
\mathbf{k}_{u} & =0 \\
\mathbf{k}_{d} & =2 \mathbf{q}_{3} \\
\mathbf{k}_{s} & =2 \mathbf{q}_{2} .
\end{aligned}
$$

We shall see this choice emerge in a different way below.

Substituting the expression (26) in Eqs. (11) and (12) and neglecting the contribution of antiparticles, the full Lagrangian reads

$$
\begin{aligned}
\mathcal{L}= & \int \frac{d \mathbf{n}}{4 \pi}\left[\psi_{\mathbf{n}, i \alpha}^{\dagger}\left(i V \cdot \partial_{i j}^{\alpha \beta}+\delta \mu_{i}(\mathbf{n}) \delta^{\alpha \beta} \delta_{i j}\right) \psi_{\mathbf{n}, \beta j}\right. \\
& +\left(\sum_{I=2}^{3} \Delta_{I} e^{i 2 \mathbf{q} \cdot \mathbf{r}} \epsilon_{I \alpha \beta} \epsilon_{I i j} \psi_{\mathbf{n}, i \alpha} C \gamma_{5} \psi_{-\mathbf{n}, \beta j} e^{-i\left(\mathbf{k}_{\mathbf{i}}+\mathbf{k}_{\mathbf{j}}\right) \cdot \mathbf{r}}\right. \\
& + \text { h.c. })]
\end{aligned}
$$

where $\delta \mu_{i}(\mathbf{n})=P_{i}^{F}-\mu-\mathbf{k}_{\mathbf{i}} \cdot \mathbf{n}$ and where the four vectors $V^{\nu}$ and $\bar{V}^{\nu}$ (the latter used only below) are defined by $V^{\nu}=(1, \mathbf{n})$ and $\bar{V}^{\nu}=(1,-\mathbf{n})$. We now see that we can get rid of the space dependence in the gap term by choosing the shift vectors $\mathbf{k}_{\mathbf{i}}$ so that they satisfy

$$
\begin{aligned}
\mathbf{k}_{\mathbf{u}}+\mathbf{k}_{\mathbf{d}} & =2 \mathbf{q}_{3} \\
\mathbf{k}_{\mathbf{u}}+\mathbf{k}_{\mathbf{s}} & =2 \mathbf{q}_{2} .
\end{aligned}
$$

Because the k's were introduced arbitrarily in the decomposition (26), the calculation could in principle be done with any choice of k's. However, eliminating the space dependence in the gap term is an enormous simplification, equivalent to yielding a propagator that is diagonal in momentum space, and is what makes the calculation tractable. So, we shall always choose k's satisfying (29). According to (29), if we choose $\mathbf{k}_{u}=0$, we recover (27). However, $\mathbf{k}_{u}$ can be chosen arbitrarily as long as $\mathbf{k}_{d}$ and $\mathbf{k}_{s}$ are then chosen to satisfy (29). This means that the choices of k's that get rid of the space dependence in the gap term are given by any combination related to (27) by adding any vector to $\mathbf{k}_{u}$ and subtracting the same vector from $\mathbf{k}_{d}$ and $\mathbf{k}_{s}$. The geometric interpretation of two examples of choices of $\mathbf{k}$ 's is described in Fig, 3 As this figure illustrates, the freedom to shift $\mathbf{k}_{u}$ while keeping $\mathbf{k}_{u}+\mathbf{k}_{d}$ and $\mathbf{k}_{u}+\mathbf{k}_{s}$ fixed corresponds to the freedom to shift integration variable, for example as we did in going from (23) to (24). In obtaining the results that we shall plot in Section V, we shall use the choice 27]; however, we have checked numerically that different choices of $\mathbf{k}_{u}$ with $\mathbf{k}_{d}$ and $\mathbf{k}_{s}$ satisfying (29) yield the same results for the gap parameter and free energy. As Fig. 4 below indicates, these different choices yield quite different intermediate stages to the calculation so the fact that we find the expected agreement between them is a nontrivial check of our numerics.

We can now employ the Nambu-Gorkov basis defined in detail in Ref. 22 given by

$$
\chi_{A}=\frac{1}{\sqrt{2}}\left(\begin{array}{c}
\psi_{\mathbf{n}} \\
C \psi_{-\mathbf{n}}^{*}
\end{array}\right)_{A},
$$

where $A=1 \ldots 9$ is a color-flavor index running over the nine quarks (three colors; three flavors) and where the $\psi_{\mathbf{n}}$ fields are defined via (26) with shift vectors chosen as in Eq. 27). In this basis, the full Lagrangian can be written in the compact form

$$
\mathcal{L}=\sum_{\mathbf{n}} \chi_{A}^{\dagger} S_{A, B}^{-1}(\mathbf{n}) \chi_{B},
$$

with

$S_{A, B}^{-1}=\left(\begin{array}{cc}\left(V \cdot \ell+\delta \mu_{A}(\mathbf{n})\right) \delta_{A B} & -\Delta_{A B} \\ -\Delta_{A B} & \left(\bar{V} \cdot \ell-\delta \mu_{A}(-\mathbf{n})\right) \delta_{A B}\end{array}\right)$,

where $\ell_{\nu}=\left(\ell_{0}, \ell_{\|} \mathbf{n}\right)$ is a four-vector. Here, $\ell_{\|}$is the "radial" momentum component of $\psi$, parallel to $\mathbf{n}$. In HDET, the momentum of a fermion is written as $\left(\mu+\ell_{\|}\right) \mathbf{n}$, with the integration over momentum space separated into an angular integration over $\mathbf{n}$ and a radial integration over $-\delta<\ell_{\|}<\delta$. Here, the cutoff $\delta$ must be smaller than $\mu$ but must be much larger than $\Delta_{0}, \delta \mu$ and $\Delta$. In the results we plot in Section $\mathrm{V}$, we shall take $\mu=500 \mathrm{MeV}, \delta=300 \mathrm{MeV}$ and $\Delta_{0}=25 \mathrm{MeV}$.

From the Lagrangian (31), following a derivation analogous to that in Ref. 12], the thermodynamic potential per unit volume can be evaluated to be

$$
\begin{aligned}
\Omega= & -\frac{\mu^{2}}{4 \pi^{2}} \sum_{a=1,18} \int_{-\delta}^{+\delta} d \ell_{\|} \int \frac{d \mathbf{n}}{4 \pi}\left|E_{a}\left(\mathbf{n}, \ell_{\|}\right)\right| \\
& +\frac{2 \Delta^{2}}{G}-\frac{\mu_{e}^{4}}{12 \pi^{2}}
\end{aligned}
$$

where we have set $\Delta_{2}=\Delta_{3}=\Delta$ and where $G$ is a coupling constant chosen in such a way that $\Delta_{0}=25 \mathrm{MeV}$ in the CFL phase found at $\mu=500 \mathrm{MeV}$ with $M_{s}=0$. In this expression, the $E_{a}$ are the energies of the quasiparticles in this phase. They are given by the 18 roots of $\operatorname{det} S^{-1}=0$, seen as an equation for $\ell_{0}$, with $S^{-1}$ the Nambu-Gorkov inverse propagator given in (32). The quasiparticle energies are functions of $\ell_{\|}$and $\mathbf{n}$, and also depend on the gap parameter $\Delta$ and the wave vectors $\mathbf{k}_{\mathbf{i}}$. The doubling of degrees of freedom in the Nambu-Gorkov formalism means that the 18 roots come in pairs whose energies are related by $E_{a}\left(\mathbf{n}, \ell_{\|}\right)=E_{b}\left(-\mathbf{n}, \ell_{\|}\right)$. One set of nine roots describes $\left(\psi_{\mathbf{n}, d}, \psi_{-\mathbf{n}, u}\right)$ and $\left(\psi_{\mathbf{n}, s}, \psi_{-\mathbf{n}, u}\right)$ pairing, while the other set describes $\left(\psi_{\mathbf{n}, u}, \psi_{-\mathbf{n}, d}\right)$ and $\left(\psi_{\mathbf{n}, u}, \psi_{-\mathbf{n}, s}\right)$ pairing. Since $\mathbf{n}$ is integrated over, the free energy can be evaluated by doing the sum in (33) over either set of nine roots, instead of over all 18, and multiplying the sum by two.

In order to determine the lowest free energy state, we need to minimize the free energy $\Omega$ given in Eq. (33) with 

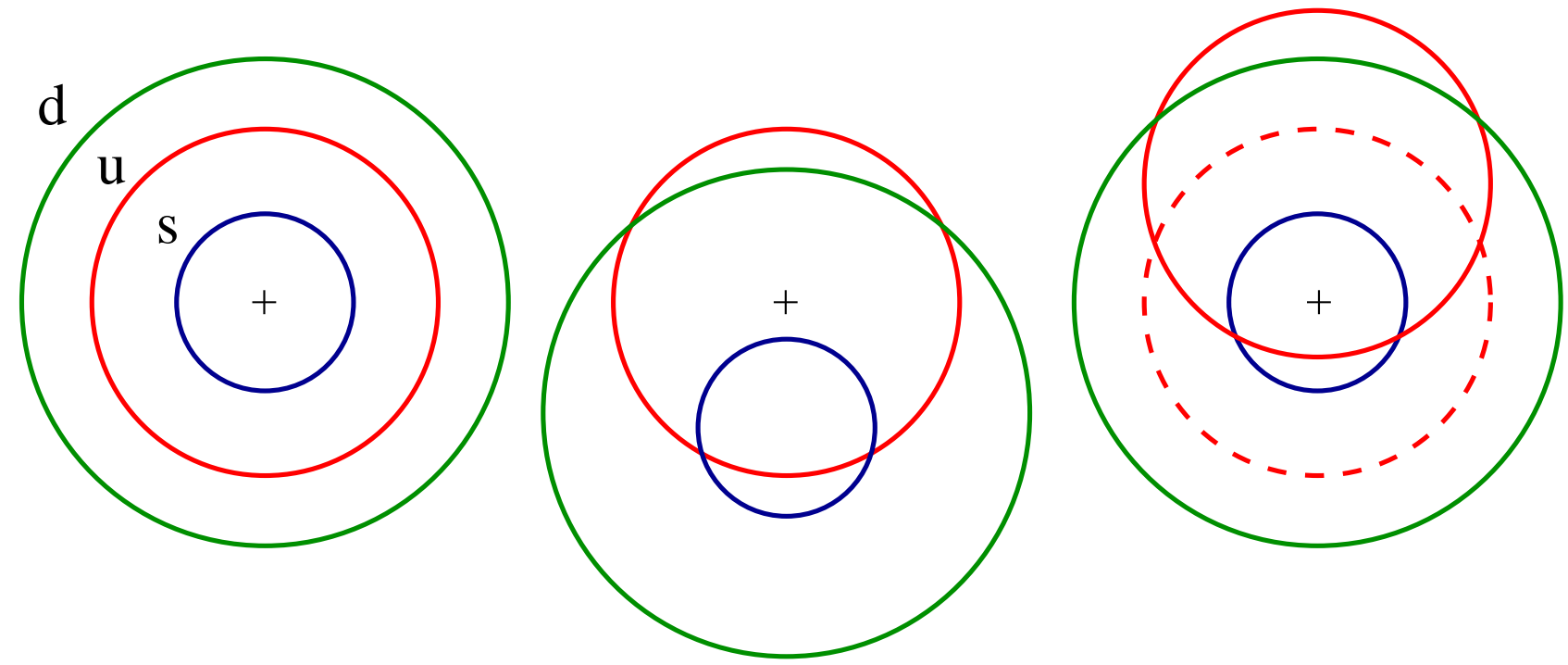

FIG. 3: Sketches showing how different choices of the shift vectors $\mathbf{k}_{\mathbf{i}}$ can be interpreted. The left panel shows unshifted $d$, $u$ and $s$ Fermi surfaces, in the absence of pairing. As in Fig. 2 we have exaggerated the magnitude of $\delta \mu / \mu$ for illustrative purposes. Now, suppose we have a condensate with $\mathbf{q}_{2}=\mathbf{q}_{3} \equiv \mathbf{q}$, with $\mathbf{q}$ a vector pointing upwards. In order to describe the $\left(\psi_{\mathbf{n}, d}, \psi_{-\mathbf{n}, u}\right)$ and $\left(\psi_{\mathbf{n}, s}, \psi_{-\mathbf{n}, u}\right)$ pairing, we shift the $u, d$ and $s$ Fermi surfaces by $-\mathbf{k}_{u},-\mathbf{k}_{d}$ and $-\mathbf{k}_{s}$ respectively (since $\psi_{\mathbf{n}, i}$ describes quarks in the vicinity of $\mu \mathbf{n}+\mathbf{k}_{i}$ ) and then reflect (i.e. take $\mathbf{n} \rightarrow-\mathbf{n}$ ) the $u$ Fermi surface. The middle panel shows the outcome if we follow this procedure with shift vectors given by (27). The $u$ Fermi surface is left unshifted (meaning its inversion is invisible in the Figure), and the $d$ and $s$ Fermi surfaces are shifted downwards by $2 \mathbf{q}$. In the right panel, we instead choose $\mathbf{k}_{u}=2 \mathbf{q}$, which according to (29) then requires $\mathbf{k}_{d}=\mathbf{k}_{s}=0$. The $d$ and $s$ Fermi surfaces are unshifted. The $u$ Fermi surface has been shifted downwards by $\mathbf{2 q}$ and then inverted, making it look as if it was shifted upwards. The location of the rings on the Fermi surfaces where pairing occurs are determined by the places where the circles cross. In both middle and right panels, the pairing rings on the $d$ and $s$ Fermi surface occur at their intersections with the $u$ Fermi surfaces whereas the pairing rings on the $u$ Fermi surface are antipodal to where these intersections appear in the Figure, since the $u$ Fermi surface was inverted in constructing the Figure. Thus, both the right and middle panels of this figure correspond to the pairing sketched in the left panel of Fig. 2 The same calculation can be done by integrating over the momentum variable of either the middle or right panel; the difference between panels is just a change of integration variable. The origins of the momentum variables are indicated by the + in each panel. Note that if we had instead chosen to describe the $\left(\psi_{\mathbf{n}, u}, \psi_{-\mathbf{n}, d}\right)$ and $\left(\psi_{\mathbf{n}, u}, \psi_{-\mathbf{n}, s}\right)$ pairing, both the middle and right panels would look inverted relative to those given but this difference also corresponds to a change of integration variable, in this case $\mathbf{n} \leftrightarrow-\mathbf{n}$.

respect to the gap parameter $\Delta$ and with respect to $\phi$, the angle between $\hat{\mathbf{q}}_{2}$ and $\hat{\mathbf{q}}_{3}$. One could also simultaneously minimize with respect to $\mu_{e}, \mu_{3}$ and $\mu_{8}$. And, one could allow $\Delta_{2} \neq \Delta_{3}$ and minimize with respect to the two gap parameters separately. However, in the results that we shall present in the next section we shall fix $\Delta_{2}=\Delta_{3}=$ $\Delta, \mu_{e}=M_{s}^{2} /(4 \mu)$ and $\mu_{3}=\mu_{8}=0$, as is correct for small $\Delta$ and as we have done in the Ginzburg-Landau analysis of Section III.

Before turning to comparing results obtained from the calculation presented in this section to those obtained with the Ginzburg-Landau approximation developed in Section III, we close this section by calculating explicitly how the free energy $\Omega$ of Eq. (33) manifests the qualitative features described in Section II and sketched in Fig. 2 with pairing occurring along ribbons of the Fermi surfaces. The easiest way to find the regions of momentum space in which pairing is important is to analyze the gap equation, obtained by varying $\Omega$ with respect to $\Delta$.
This takes the form

$$
\Delta \propto \int_{-\delta}^{+\delta} d \ell_{\|} \int d \mathbf{n} f\left(\mathbf{n}, \ell_{\|}\right)
$$

with

$$
f\left(\mathbf{n}, \ell_{\|}\right)=\sum_{a=1}^{9} \frac{\partial E_{a}}{\partial \Delta} \operatorname{Sign}\left(E_{a}\right) .
$$

(Either set of nine quasiparticle energies could be chosen, but to make the comparison with Fig. 3 we have used those describing $\left(\psi_{\mathbf{n}, d}, \psi_{-\mathbf{n}, u}\right)$ and $\left(\psi_{\mathbf{n}, s}, \psi_{-\mathbf{n}, u}\right)$ pairing.) In Fig. (4) we plot $f$, the integrand in the gap equation, as a function of $\ell_{\|}$and $\cos \theta$ where $\theta$ is the polar angle specified by $\mathbf{n}$. (A plot of the integrand in the expression for $\Omega$ in Eq. (33) evaluated with $\Delta$ minus that evaluated with $\Delta=0$ yields a very similar figure.) We have plotted $f\left(\cos \theta, \ell_{\|}\right)$for two different choices of the shift vectors $\mathbf{k}_{i}$, 

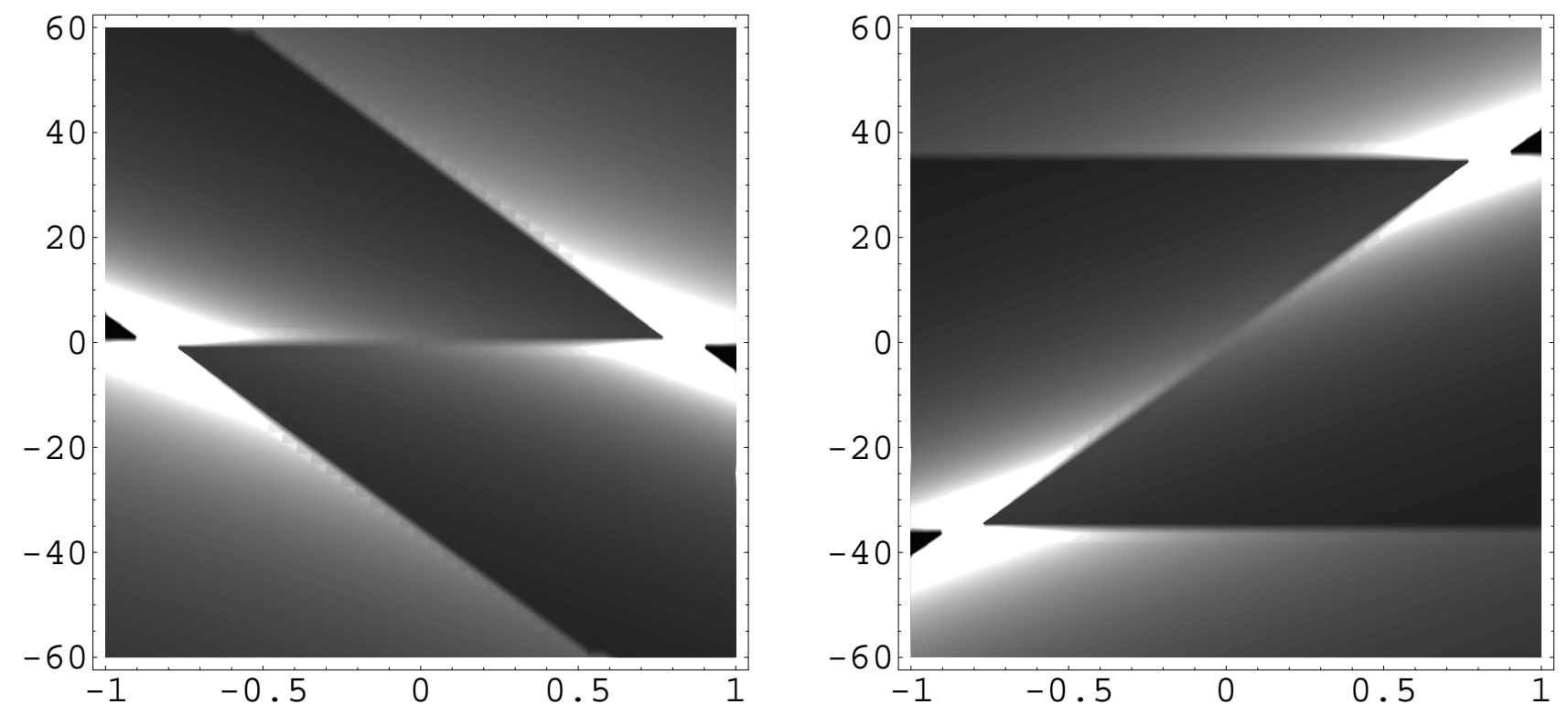

FIG. 4: Gap equation integrand $f$, defined in Eq. 35, as a function of momentum. In each panel, the horizontal axis is $\cos \theta$ where $\theta$ is the polar angle specified by $\mathbf{n}$, the vertical axis is $\ell_{\|}$in $\mathrm{MeV}$, and the grey scale indicates the value of $f$ : black corresponds to $f=0$ and white to the largest values of $f$. In both panels, $\mu=500 \mathrm{MeV}$ and $\delta \mu=17.7 \mathrm{MeV}$, which is $0.707 \Delta_{0}$ for $\Delta_{0}=25 \mathrm{MeV}$, the interaction strength that we shall use in the next section. In both panels, $\mathbf{q}_{2}=\mathbf{q}_{3} \equiv \mathbf{q}$ with $q=1.20 \delta \mu=21.2 \mathrm{MeV}$ and $\mathbf{q}$ pointing in the $z$-direction. And, in both panels $\Delta=1 \mathrm{MeV}$. In the left panel, $\mathbf{k}_{u}=0$ and $\mathbf{k}_{d}=\mathbf{k}_{s}=2 \mathbf{q}$ as in the middle panel of Fig. 3. The unshifted $u$ Fermi surface is centered in momentum space, meaning that it appears in the left panel as a horizontal line at $\ell_{\|}=0$. The shifted $d$ and $s$ Fermi surfaces appear as diagonal lines, with the shifted $d$ Fermi surface inside the $u$ Fermi surface at the north pole $(\cos \theta=1)$ and the shifted $s$ Fermi surface outside the $u$ Fermi surface at the south pole. In the right panel, $\mathbf{k}_{u}=2 \mathbf{q}$ and $\mathbf{k}_{d}=\mathbf{k}_{s}=0$ as in the right panel of Fig. 3 The unshifted $d$ and $s$ Fermi surfaces are centered in momentum space, meaning that they appear in the right panel as horizontal lines at $\ell_{\|}= \pm 2 \delta \mu$. (Note that $\ell_{\|}$is measured relative to where the unshifted $u$-Fermi surface would have been, shown as a dashed circle in the right panel of Fig. [3 that corresponds to $\ell_{\|}=0$ in the right panel here.) The shifted $u$ Fermi surface appears in the right panel here as a diagonal line, outside the $d$ Fermi surface at the north pole and inside the $s$ Fermi surface at the south pole. Pairing is most important in the bright white regions, centered where the shifted Fermi surfaces cross. The sketches provided in Fig. 2] and particularly in Fig. 3 serve to help visualize the "momentum-space geometry" and pairing regions depicted in the present figure.

corresponding to the middle and right panels in Fig. 3 The differences between the two panels of Fig. 4 come entirely from the different choices of shift vectors; both panels correspond to the same condensate, with $\mathbf{q}_{2} \| \mathbf{q}_{\mathbf{3}}$ as in the left panel of Fig. 2 And, we find excellent agreement when we integrate $f$ depicted in either the left or the right panel of Fig. 4 to obtain the right-hand side of the gap equation (34), and similar agreement when we do the integral in Eq. (33) needed to evaluate the free energy $\Omega$ with either choice of shift vectors.

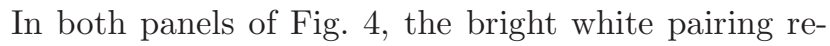
gions near where the shifted and unshifted Fermi surfaces cross are clearly visible, as are the jet black blocking regions near the north and south poles of the Fermi surfaces at $\cos \theta= \pm 1$ where no pairing occurs. The pairing regions are centered at $\theta=67.1^{\circ} / 2$ and $\theta=180^{\circ}-67.1^{\circ} / 2$, corresponding to $\cos \theta= \pm 0.833$. The dark but not black regions between Fermi surfaces are places where either $u-d$ or $u$-s pairing is blocked, but the other is allowed. Note that even though the formal pairing regions (regions where $f \neq 0$ ) extend far from the Fermi surfaces, the bright white regions where the maximal value of $f$ is attained are localized near where Fermi surfaces cross. So far, all as expected.

Even with a rather small value of $\Delta-$ in Fig. 4 $\Delta / \delta \mu=0.056$ - the ribbons on the Fermi surfaces where pairing occurs do not look very narrow. In fact, if we increase $\Delta$ to the point at which $\Delta / \delta \mu=0.19$, keeping all other parameters as in Fig. 4 the blocking regions at the north and south poles visible in Fig. 团disappear entirely. The pairing "ribbons" become so wide that the ribbon centered at $\theta=67.1^{\circ}$ expands to encompass $\theta=0$, becoming more of a hat for the Fermi surface than a ribbon on it. Furthermore, even in Fig. 4 where the pairing rib- 

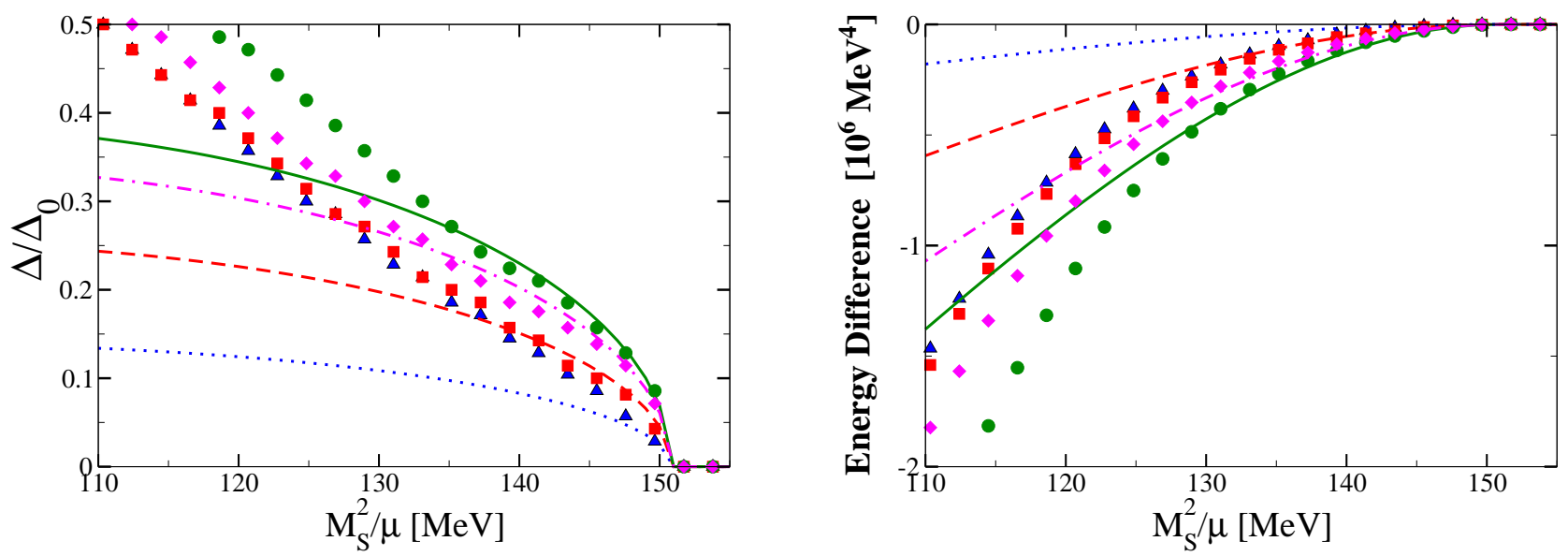

FIG. 5: Plot of $\Delta / \Delta_{0}$ (left panel) and of the free energy relative to neutral non interacting quark matter (right panel) as a function of $M_{s}^{2} / \mu$ for four values of the angle $\phi$ between $\mathbf{q}_{2}$ and $\mathbf{q}_{3}$. The various lines correspond to the calculations done in the Ginzburg-Landau approximation described in Section III whereas dots correspond to the NJL calculation of Section IV, done without making a Ginzburg-Landau approximation. The full lines (green online) and circles correspond to $\phi=0$, the dashed-dotted lines (magenta online) and diamonds correspond to $\phi=2 \pi / 3$, the dashed lines (red online) and squares correspond to $\phi=7 \pi / 8$, the dotted lines (blue online) and triangles correspond to $\phi=31 \pi / 32$.

bons on the Fermi surfaces are somewhat narrow, in that there are blocking regions at the poles, these blocking regions are surrounded by regions of momentum space where pairing is quite significant. Just a little distance in $\ell_{\|}$away from the Fermi surfaces, the angular extent of the regions where pairing is significant grows rapidly, becoming much wider than right at the Fermi surfaces themselves. These are all indications that even though the Ginzburg-Landau approximation is formally controlled by the parameter $\Delta / \delta \mu$, it may break down quantitatively at rather small values of $\Delta / \delta \mu$. After all, in the Ginzburg-Landau limit $\Delta / \delta \mu \rightarrow 0$ the pairing is dominated by infinitesimally narrow ribbons exactly where the shifted Fermi surfaces cross. Using this as a basis for approximation cannot yield even a qualitative description of the physics once $\Delta / \delta \mu \sim 0.2$, as even with this small a value of $\Delta / \delta \mu$ the regions where pairing is significant are no longer confined to narrow ribbons but have spread over considerable regions of the Fermi surfaces. Indeed, the extent of the bright white regions where pairing is

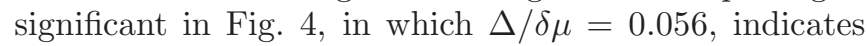
that the Ginzburg-Landau approximation may cease to be quantitatively reliable at values of $\Delta / \delta \mu$ below 0.2 .

\section{COMPARISONS AND CONCLUSIONS}

In Fig. 5 we compare our results for the gap parameter and the free energy in the crystalline color superconducting phase calculated in the Ginzburg-Landau approximation of Section III with those obtained without making this approximation in Section IV. We have done the calculations with $\mu=500 \mathrm{MeV}$ and a coupling strength chosen so that $\Delta_{0}$, the BCS gap in the CFL phase at $M_{s}=0$, is $25 \mathrm{MeV}$. We vary $M_{s}$, but plot quantities versus $M_{s}^{2} / \mu$ because the most important effect of nonzero $M_{s}$ is the splitting between the $d, u$ and $s$ Fermi momenta given in Eq. (7), controlled by $\delta \mu=M_{s}^{2} /(8 \mu)$. We analyze the condensate (11) with $\Delta_{2}=\Delta_{3} \equiv \Delta$ and $q_{2}=q_{3} \equiv q$. At each value of $M_{s}^{2} / \mu$, we choose $q=\eta \delta \mu=\eta M_{s}^{2} /(8 \mu)$, where $\eta=1.1997$. We fix $\mu_{3}=\mu_{8}=0$ and $\mu_{e}=M_{s}^{2} /(4 \mu)$, as appropriate for neutral three-flavor crystalline quark matter with $\Delta / \delta \mu \ll 1$, for which the Ginzburg-Landau approximation is valid. We leave investigating the extent to which these chemical potentials may shift at larger $\Delta$ to future work. We show our results for four values of the angle between $\mathbf{q}_{2}$ and $\mathbf{q}_{3}: \phi=0,2 \pi / 3,7 \pi / 8$ and $31 \pi / 32$. The lines correspond to the Ginzburg-Landau analysis of Section III, where we have plotted $\Delta$ and $\Omega-\Omega_{n}$ of Eqs. (19) and (20), using Eqs. (15), (16), and (17) and using Eq. (14) to relate $\alpha$ to $\delta \mu$ and hence to $M_{s}^{2} / \mu$. The points correspond to the NJL calculation of Section IV, where we have minimized $\Omega$ of Eq. (33) with respect to $\Delta$.

We see in Fig. 5 that the NJL calculation has a second order transition at $M_{s}^{2} / \mu \sim 151 \mathrm{MeV}$, corresponding to $\delta \mu \sim 0.754 \Delta_{0}$, for all values of the angle $\phi$. This result is in agreement with the Ginzburg-Landau calculation, in which the location of the phase transition depends only on $\alpha$, which is independent of $\phi$. We then see that near the phase transition, where $\Delta / \Delta_{0}$ and hence $\Delta / \delta \mu$ are small, we find good agreement between the NJL calculation and the Ginzburg-Landau approximation, as expected. For all values of $\phi$, as $\Delta / \Delta_{0}$ increases as $M_{s}^{2} / \mu$ is decreased farther from the transition, we see that both $\Delta$ and $\left|\Omega-\Omega_{n}\right|$ increase more rapidly with decreasing 
$M_{s}^{2} / \mu$ than predicted by the Ginzburg-Landau calculation. When the Ginzburg-Landau approximation breaks down, it does so conservatively, underpredicting both $\Delta$ and $\left|\Omega-\Omega_{n}\right|$ for the entire one parameter family of "crystal structures" parameterized by $\phi$. (This behavior also occurs in the two-flavor model with condensate (9) 36].) Furthermore, even where the Ginzburg-Landau approximation has broken down quantitatively, it correctly predicts the qualitative feature that at all values of $M_{s}^{2} / \mu$ the most favorable crystal structure is that with $\phi=0$. As we saw in the discussion of Fig. 2 this can be attributed at least qualitatively to the fact that for $\mathbf{q}_{2} \| \mathbf{q}_{3}$ the two pairing ribbons on the $u$-Fermi surface are farthest apart. We see that the Ginzburg-Landau approximation is useful as a qualitative guide even where it has broken down quantitatively.

It is evident from Fig. 5 that the extent of the regime in which the Ginzburg-Landau approximation is quantitatively reliable is strongly $\phi$-dependent. In the best case, which it turns out is $\phi=0$, the results of the GinzburgLandau calculation are in good agreement with those of the full NJL calculation as long as $\Delta / \Delta_{0} \lesssim 0.25$, corresponding to $\Delta / \delta \mu \lesssim 0.35$. On the one hand, this looks like a somewhat small value of $\Delta / \delta \mu$. However, it is remarkable that the Ginzburg-Landau approximation works so well for this large a value of $\Delta / \delta \mu$ : after all, we saw in the discussion of Fig. 4 in Section IV that for $\phi=0$ even with $\Delta / \delta \mu$ only 0.19 the pairing "ribbons" that characterize the Ginzburg-Landau approximation have broadened into "pairing hats" encompassing the north and south poles of the Fermi surfaces. For larger $\phi$, the Ginzburg-Landau approximation yields quantitatively reliable results only for much smaller $\Delta$. For example, with $\phi=31 \pi / 32$ we have zoomed in on the region near the second order phase transition and seen that the Ginzburg-Landau calculation does give results in quantitative agreement with the full NJL calculation, but only for $\Delta / \Delta_{0} \lesssim 0.04$, corresponding to $\Delta / \delta \mu \lesssim 0.05$. Why does the regime of quantitative validity of the GinzburgLandau approximation shrink with increasing $\phi$ ? One possible qualitative explanation is related to the behavior of the pairing regions displayed for $\phi=0$ in Fig. 4 We saw in that figure that already at quite small $\Delta / \delta \mu$, pairing is significant over wide swaths of angle in momentum space, particularly a little away from the Fermi surfaces. Since at $\phi=\pi$ the two pairing regions on the $u$ Fermi surface overlap completely, the closer $\phi$ gets to $\pi$ the smaller the value of $\Delta / \delta \mu$ needed for the spreading of the pairing regions to become comparable to the separation between pairing regions. This qualitative argument does suggest that the Ginzburg-Landau approximation should break down at smaller $\Delta / \delta \mu$ for larger $\phi$, as we find. It does not explain why this breakdown always results in $\Delta$ being enhanced relative to that predicted by the Ginzburg-Landau approximation.

A quantitative study of the radius of convergence of the Ginzburg-Landau approximation would require evaluating (at least) the $\Delta^{6}$ terms, whose coefficients we shall generically call $\gamma$. Because we are working in the vicinity of a point where $\alpha=0$, the first estimator of the radius of convergence that we can construct comes by requiring $\gamma \Delta^{6} \lesssim\left(\beta+\beta_{23}\right) \Delta^{4}$. The results of the comparison in Fig. 5]are not conclusive on this point, but they certainly indicate that the radius of convergence in $\Delta$ decreases with increasing $\phi$, decreasing towards zero for $\phi \rightarrow \pi$. Given that we see from Fig. 1 that $\beta_{23}$ (and hence $\beta+\beta_{23}$ ) increases with $\phi$ and diverges for $\phi \rightarrow \pi$, the apparent behavior of the radius of convergence in Fig. 5] suggests that $\gamma$ should diverge even faster for $\phi \rightarrow \pi$.

In Fig. 6] we compare our results for the free energy of the three-flavor crystalline color superconductor with the most favorable "crystal" structure that we have found, namely that with $\mathbf{q}_{2} \| \mathbf{q}_{3}$, to the free energy of the CFL and gapless CFL phases [11, 12, 15]. Recall that, as discussed in Section I, the gapless CFL phase is known to be unstable with respect to counterpropagating currents 22]. This means that at every value of $M_{s}^{2} / \mu$ where the CFL phase has been superseded by the gCFL phase and where the gCFL phase has lower free energy than unpaired quark matter, there must be one or more phases with free energy below that of the gCFL phase. We see that the crystalline phase we have analyzed, with its particularly simple crystal structure consisting of only two plane waves, one for the $\langle u d\rangle$ condensate and one for the $\langle u s\rangle$ condensate, has a lower free energy than both the gCFL and unpaired phases in a window $128 \mathrm{MeV}<$ $M_{s}^{2} / \mu<151 \mathrm{MeV}$. Recalling that the Ginzburg-Landau approximation is reliable over the widest domain precisely for the case with $\mathbf{q}_{2} \| \mathbf{q}_{3}$, we note from Fig. 5 that the Ginzburg-Landau analysis provides good approximation to the results of the full NJL calculation within the window $128 \mathrm{MeV}<M_{s}^{2} / \mu<151 \mathrm{MeV}$. Hence, our result for the extent of this window agrees with that in Ref. 37], which was obtained in the Ginzburg-Landau approximation.

We do not actually expect the condensate we have analyzed to be the stable, lowest free energy, phase. Instead, we expect that, as in the investigation of two-flavor crystalline color superconductivity in Ref. [36], crystal structures with more plane waves will yield significantly lower free energy. It will be interesting to investigate which crystal structures are most favorable, and over what regime of $M_{s}^{2} /(4 \mu)$ they have lower free energy than the gCFL phase. Looking ahead to such an investigation, the implications of our tests of the Ginzburg-Landau approximation in the simple case in which we have been able to calculate both without and with this approximation can be seen as either a glass half empty or a glass half full. On the one hand, we find that the approximation is quantitatively reliable only for values of $\Delta / \delta \mu$ that are small and for some crystal structures (those with $\mathbf{q}_{2}$ and $\mathbf{q}_{3}$ close to antiparallel) very small. This means that if there are robust crystal structures with large gaps and condensation energies, they may not be described reliably within the Ginzburg-Landau approximation. On the other hand, we find that even when it breaks down 


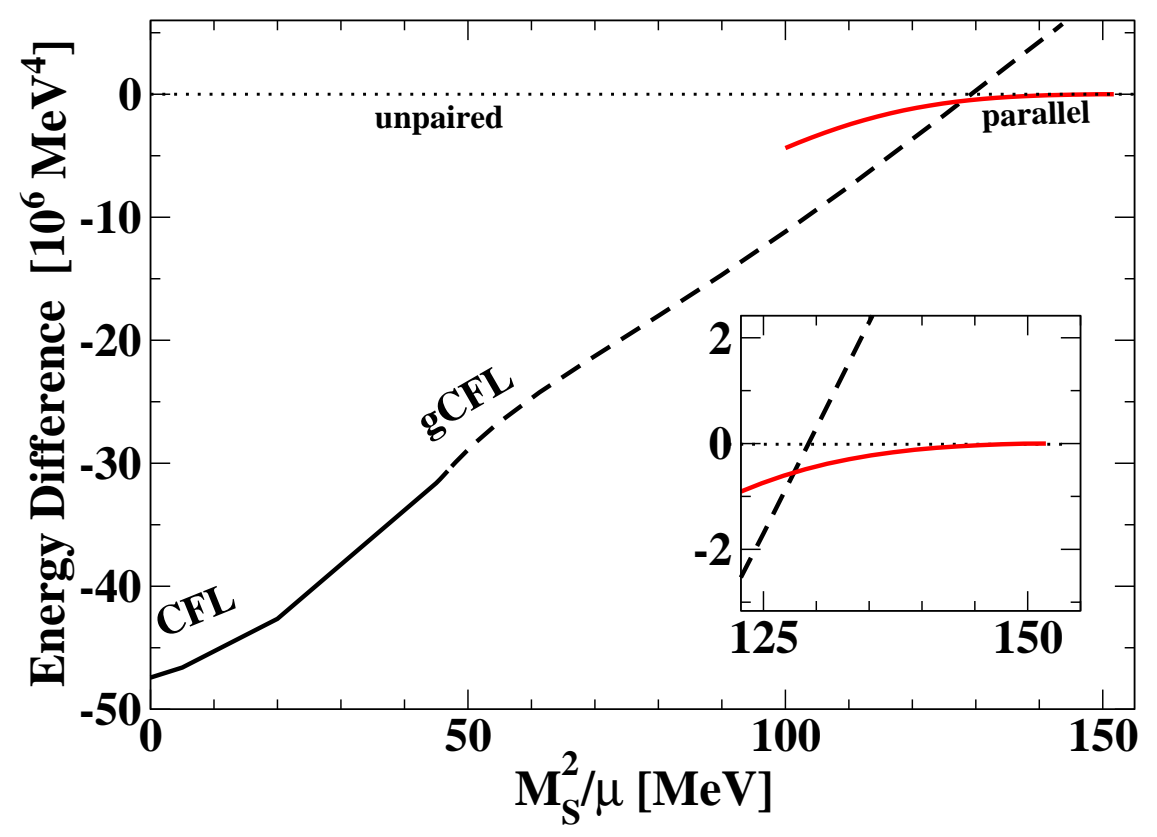

FIG. 6: Plot of the free energy of the three-flavor crystalline color superconducting phase with condensate (11) with $\Delta_{1}=0$, $\Delta_{2}=\Delta_{3}$, and $\mathbf{q}_{2} \| \mathbf{q}_{3}$, compared to the free energy of the CFL and gapless CFL phases. As throughout, we are normalizing the interaction to yield a CFL gap parameter at $M_{s}=0$ of $\Delta_{0}=25 \mathrm{MeV}$. We have plotted the free energy calculated as in Section IV, without making a Ginzburg-Landau approximation. We see that this crystalline phase, with its particularly simple "crystal" structure, is favored over the other phases shown for $128 \mathrm{MeV}<M_{s}^{2} / \mu<151 \mathrm{MeV}$.

quantitatively the Ginzburg-Landau approximation remains useful as a qualitative guide, correctly predicting that the favored "crystal" structure among our one parameter family of possibilities is that with $\mathbf{q}_{2} \| \mathbf{q}_{3}$. Furthermore, the cases in which the domain of reliability of the approximation is smallest, those with $\mathbf{q}_{2}$ and $\mathbf{q}_{3}$ close to antiparallel, are also the cases with the least favorable free energy. This suggests that when more complicated crystal structures are analyzed, among them the domain in which the Ginzburg-Landau approximation is reliable may prove wider for those structures which are more favorable (i.e. have lower free energy). As an example, recall that in our analysis we found narrow domains of reliability for the Ginzburg-Landau approximation for $\phi$ near $\pi$, the same regime where we found that the condensate we analyzed was anyway not favored because it had a larger free energy than one in which only two flavors of quarks pair. Finally, in all the cases where we have been able to test it, when the Ginzburg-Landau approximation breaks down it does so conservatively, underpredicting the magnitude of $\Delta$ and the favorability of the free energy. We conclude that there is strong motivation to explore more complicated and realistic crystal structures at least initially using this approximation.

\section{Acknowledgement}

We thank R. Gatto, E. Gubankova, G. Nardulli, M. Ruggieri and A. Schmitt for useful discussions. The work of MM has been supported by the "Bruno Rossi" fellowship program. KR and RS acknowledge the hospitality of the Nuclear Theory Group at LBNL. This research was supported in part by the Office of Nuclear Physics of the Office of Science of the U.S. Department of Energy under contract \#DE-AC02-05CH11231 and cooperative research agreement \#DF-FC02-94ER40818.
[1] For reviews, see K. Rajagopal and F. Wilczek, arXiv:hep-ph/0011333 M. G. Alford, Ann. Rev. Nucl. Part. Sci. 51, 131 (2001) arXiv:hep-ph/0102047; G. Nardulli, Riv. Nuovo Cim. 25N3, 1 (2002) arXiv:hep-ph/0202037; S. Reddy, Acta Phys. Polon. B 33, 4101 (2002) arXiv:nucl-th/0211045]; T. Schäfer,
arXiv:hep-ph/0304281 D. H. Rischke, Prog. Part. Nucl. Phys. 52, 197 (2004) arXiv:nucl-th/0305030; M. Alford, Prog. Theor. Phys. Suppl. 153, 1 (2004) arXiv:nucl-th/0312007; M. Buballa, Phys. Rept. 407, 205 (2005) arXiv:hep-ph/0402234; H. c. Ren, arXiv:hep-ph/0404074 I. 
Shovkovy, arXiv:nucl-th/0410091 T. Schäfer, arXiv:hep-ph/0509068

[2] J. Bardeen, L.N. Cooper, and J.R. Schrieffer, Phys. Rev. 108, 1175 (1957).

[3] M. Iwasaki and T. Iwado, Phys. Lett. B 350, 163 (1995). T. Schäfer, Phys. Rev. D 62, 094007 (2000) arXiv:hep-ph/0006034; M. Buballa, J. Hosek and M. Oertel, Phys. Rev. Lett. 90, 182002 (2003) arXiv:hep-ph/0204275; A. Schmitt, Q. Wang and D. H. Rischke, Phys. Rev. D 66, 114010 (2002) arXiv:nucl-th/0209050; M. G. Alford, J. A. Bowers, J. M. Cheyne and G. A. Cowan, Phys. Rev. D 67, 054018 (2003) arXiv:hep-ph/0210106; A. Schmitt, Phys. Rev. D 71, 054016 (2005) arXiv:nucl-th/0412033.

[4] M. G. Alford, K. Rajagopal and F. Wilczek, Phys. Lett. B 422, 247 (1998) arXiv:hep-ph/9711395.

[5] M. G. Alford, K. Rajagopal and F. Wilczek, Nucl. Phys. B 537, 443 (1999) arXiv:hep-ph/9804403.

[6] M. Alford and K. Rajagopal, JHEP 0206, 031 (2002) arXiv:hep-ph/0204001.

[7] K. Iida and G. Baym, Phys. Rev. D 63, 074018 (2001) [Erratum-ibid. D 66, 059903 (2002)] arXiv:hep-ph/0011229;

[8] Stable bulk matter must be neutral under all gauged charges, whether they are spontaneously broken or not. In the case of the electromagnetic gauge symmetry, this simply requires zero charge density. In the case of the color gauge symmetry, bulk matter must in fact be a color singlet, which is a more restrictive condition than mere color neutrality. However, the free energy cost of projecting a color neutral state onto a color singlet state falls rapidly with volume, as long as we are considering volumes larger than the size of a Cooper pair. Given that if quark matter occurs within the core of a neutron star the relevant volumes will be of order cubic kilometers, whereas Cooper pairs have sizes of order fm, it is more than sufficient to consider only the consequences of enforcing color neutrality. See P. Amore, M. C. Birse, J. A. McGovern and N. R. Walet, Phys. Rev. D 65, 074005 (2002) arXiv:hep-ph/0110267.

[9] A. W. Steiner, S. Reddy and M. Prakash, Phys. Rev. D 66, 094007 (2002) arXiv:hep-ph/0205201.

[10] M. Huang, P. f. Zhuang and W. q. Chao, Phys. Rev. D 67, 065015 (2003) arXiv:hep-ph/0207008; F. Neumann, M. Buballa and M. Oertel, Nucl. Phys. A 714, 481 (2003) arXiv:hep-ph/0210078.

[11] M. Alford, C. Kouvaris and K. Rajagopal, Phys. Rev. Lett. 92, 222001 (2004) arXiv:hep-ph/0311286.

[12] M. Alford, C. Kouvaris and K. Rajagopal, Phys. Rev. D 71, 054009 (2005) arXiv:hep-ph/0406137.

[13] M. Alford, C. Kouvaris and K. Rajagopal, arXiv:hep-ph/0407257

[14] S. B. Rüster, I. A. Shovkovy and D. H. Rischke, Nucl. Phys. A 743, 127 (2004) arXiv:hep-ph/0405170.

[15] K. Fukushima, C. Kouvaris and K. Rajagopal, Phys. Rev. D 71, 034002 (2005) arXiv:hep-ph/0408322.

[16] M. Alford, P. Jotwani, C. Kouvaris, J. Kundu and K. Rajagopal, Phys. Rev. D 71, 114011 (2005) arXiv:astro-ph/0411560.

[17] H. Abuki, M. Kitazawa and T. Kunihiro, Phys. Lett. B 615, 102 (2005) arXiv:hep-ph/0412382.

[18] S. B. Rüster, V. Werth, M. Buballa, I. A. Shovkovy and D. H. Rischke, Phys. Rev. D 72, 034004 (2005) arXiv:hep-ph/0503184.
[19] I. Shovkovy and M. Huang, Phys. Lett. B 564, 205 (2003) arXiv:hep-ph/0302142; M. Huang and I. Shovkovy, Nucl. Phys. A 729, 835 (2003) arXiv:hep-ph/0307273.

[20] E. Gubankova, W. V. Liu and F. Wilczek, Phys. Rev. Lett. 91, 032001 (2003).

[21] M. Huang and I. A. Shovkovy, Phys. Rev. D 70, 051501 (2004) arXiv:hep-ph/0407049; M. Huang and I. A. Shovkovy, Phys. Rev. D 70, 094030 (2004) arXiv:hep-ph/0408268; I. Giannakis and H. C. Ren, Phys. Lett. B 611, 137 (2005) arXiv:hep-ph/0412015; M. Alford and Q. h. Wang, J. Phys. G 31, 719 (2005) arXiv:hep-ph/0501078; M. Huang, Phys. Rev. D 73, 045007 (2006) arXiv:hep-ph/0504235.

[22] R. Casalbuoni, R. Gatto, M. Mannarelli, G. Nardulli and M. Ruggieri, Phys. Lett. B 605, 362 (2005) [Erratumibid. B 615, 297 (2005)] arXiv:hep-ph/0410401; K. Fukushima, Phys. Rev. D 72, 074002 (2005) arXiv:hep-ph/0506080.

[23] P. F. Bedaque, H. Caldas and G. Rupak, Phys. Rev. Lett. 91, 247002 (2003) arXiv:cond-mat/0306694; H. Caldas, Phys. Rev. A 69, 063602 (2004) arXiv:hep-ph/0312275; M. M. Forbes, E. Gubankova, W. V. Liu and F. Wilczek, Phys. Rev. Lett. 94, 017001 (2005) arXiv:hep-ph/0405059; J. Carlson and S. Reddy, Phys. Rev. Lett. 95, 060401 (2005) arXiv:cond-mat/0503256.

[24] M.W. Zwierlein, A. Schirotzek, C.H. Schunck, and W. Ketterle, Science 311, 492 (2006) arXiv:cond-mat/0511197.

[25] G.B. Partridge, W. Li, R.I. Kamar, Y.-a. Liao, and R.G. Hulet, Science 311, 503 (2006) arXiv:cond-mat/0511752.

[26] D. Bailin and A. Love, Phys. Rept. 107, 325 (1984).

[27] R. Rapp, T. Schäfer, E. V. Shuryak and M. Velkovsky, Phys. Rev. Lett. 81, 53 (1998) arXiv:hep-ph/9711396.

[28] K. Rajagopal and A. Schmitt, Phys. Rev. D 73, 045003 (2006) arXiv:hep-ph/0512043.

[29] M. G. Alford, J. A. Bowers and K. Rajagopal, Phys. Rev. D 63, 074016 (2001) arXiv:hep-ph/0008208;

[30] J. .A. Bowers, J. Kundu, K. Rajagopal and E. Shuster, Phys. Ref. D 64, 014024 (2001) arXiv:hep-ph/0101067;

[31] R. Casalbuoni, R. Gatto, M. Mannarelli and G. Nardulli, Phys. Lett. B 511, 218 (2001) arXiv:hep-ph/0101326.

[32] A. K. Leibovich, K. Rajagopal and E. Shuster, Phys. Rev. D 64, 094005 (2001) arXiv:hep-ph/0104073;

[33] J. Kundu and K. Rajagopal, Phys. Rev. D 65, 094022 (2002) arXiv:hep-ph/0112206;

[34] R. Casalbuoni and G. Nardulli, Rev. Mod. Phys. 263, 320 (2004) arXiv:hep-ph/0305069;

[35] R. Casalbuoni, M. Ciminale, M. Mannarelli, G. Nardulli, M. Ruggieri and R. Gatto, Phys. Rev. D 70, 054004 (2004) arXiv:hep-ph/0404090.

[36] J. A. Bowers and K. Rajagopal, Phys. Rev. D 66, 065002 (2002) arXiv:hep-ph/0204079.

[37] R. Casalbuoni, R. Gatto, N. Ippolito, G. Nardulli and M. Ruggieri, Phys. Lett. B 627, 89 (2005) arXiv:hep-ph/0507247.

[38] M. Ciminale, G. Nardulli, M. Ruggieri and R. Gatto, arXiv:hep-ph/0602180

[39] A. I. Larkin and Yu. N. Ovchinnikov, Zh. Eksp. Teor. Fiz. 47, 1136 (1964)[Sov. Phys. JETP 20, 762 (1965)]; P. Fulde and R. A. Ferrell, Phys. Rev. 135, A550 (1964); S. Takada and T. Izuyama, Prog. Theor. Phys. 41, 635 (1969).

[40] P. F. Bedaque and T. Schäfer, Nucl. Phys. A 697, 
802 (2002) arXiv:hep-ph/0105150; D. B. Kaplan and S. Reddy, Phys. Rev. D 65, 054042 (2002) arXiv:hep-ph/0107265; A. Kryjevski, D. B. Kaplan and T. Schäfer, Phys. Rev. D 71, 034004 (2005) arXiv:hep-ph/0404290;

[41] A. Kryjevski and T. Schäfer, Phys. Lett. B 606, 52 (2005) arXiv:hep-ph/0407329; A. Kryjevski and D. Yamada, Phys. Rev. D 71, 014011 (2005) arXiv:hep-ph/0407350. M. Buballa, Phys. Lett. B 609, 57 (2005) arXiv:hep-ph/0410397; M. M. Forbes, Phys. Rev. D 72, 094032 (2005) arXiv:hep-ph/0411001.

[42] A. Kryjevski, arXiv:hep-ph/0508180 T. Schafer, Phys. Rev. Lett. 96, 012305 (2006) arXiv:hep-ph/0508190; see also D. K. Hong, arXiv:hep-ph/0506097

[43] D. K. Hong, Phys. Lett. B 473, 118 (2000) arXiv:hep-ph/9812510; D. K. Hong, Nucl. Phys. B 582, 451 (2000) arXiv:hep-ph/9905523; S. R. Beane, P. F. Bedaque and M. J. Savage, Phys. Lett. B 483, 131 (2000) arXiv:hep-ph/0002209; R. Casal- buoni, R. Gatto and G. Nardulli, Phys. Lett. B 498, 179 (2001) [Erratum-ibid. B 517, 483 (2001)] arXiv:hep-ph/0010321; R. Casalbuoni, R. Gatto, M. Mannarelli and G. Nardulli, Phys. Lett. B 524, 144 (2002) arXiv:hep-ph/0107024; T. Schafer, Phys. Rev. D 65, 074006 (2002) arXiv:hep-ph/0109052. T. Schafer, Nucl. Phys. A 728, 251 (2003) arXiv:hep-ph/0307074.

[44] G. Nardulli, in Ref. 1].

[45] A. Gerhold and A. Rebhan, Phys. Rev. D 68, 011502 (2003) arXiv:hep-ph/0305108; A. Kryjevski, Phys. Rev. D 68, 074008 (2003) arXiv:hep-ph/0305173; A. Gerhold, Phys. Rev. D 71, 014039 (2005) arXiv:hep-ph/0411086. D. D. Dietrich and D. H. Rischke, Prog. Part. Nucl. Phys. 53, 305 (2004) arXiv:nucl-th/0312044;

[46] K. Rajagopal and F. Wilczek, Phys. Rev. Lett. 86, 3492 (2001) arXiv:hep-ph/0012039. 